CERN - PS DIVISION

CERN/PS 2000-071 (BD)

8 November 2000

\title{
TRANSVERSE PROFILE MONITOR USING ION PROBE BEAMS
}

\author{
J. Bosser, C. Dimopoulou, A. Feschenko ${ }^{1)}$ and R. Maccaferri
}

\begin{abstract}
A profile monitor is described that makes use of a low-intensity and low-energy ion beam to measure the transverse profile of a dense proton beam of small dimensions. Three techniques are considered based on the use of ion beams having a pencil, curtain, or cylindrical shape. The detector is almost non-interceptive for the proton beam and does not introduce disturbances in the machine environment. The theoretical aspects of the techniques used, together with experimental results obtained at the CERN SPS and Linac, are presented.
\end{abstract}

Submitted to Nuclear Instruments and Methods in Physics Research

1) Present address: Institute for Nuclear Research, Moscow. 
The aim of this detector is to measure the transverse distribution of small-dimension, high-density particle beams. The principle on which the detector is based has been reported in Refs. [1]. It relies on the deflection of a low-energy, pencil-beam of ions passing perpendicularly through a dense particle beam. In the present paper, the analysed (or probed) beam consists of protons. The proof of principle was reported in Ref. [2a], after experiments made on the CERN SPS beam, and in Ref. [2b].

In addition to the pencil-beam technique, two further methods are also considered. They are based on the use of an 'ion curtain' [2a], [3] and on the 'shadowing' effect induced by the analysed beam [2a], [4] on a cylindrical probe beam.

All three methods are practically non-interceptive and do not perturb the machine environment, in particular the vacuum.

After a brief description of the principles, a summary of the set-up and of the measurements made at the CERN SPS will be given. Though it was initially intended to measure LHC-type beams, we shall also mention the possible use of such an ion 'profilometer' on other machines.

\section{DATA AND SYMBOLS}

\subsection{Data}

$q=1.6 \cdot 10^{-19} \mathrm{C}$, elementary charge;

$\epsilon_{0}=8.854 \cdot 10^{-12} \mathrm{~F} \mathrm{~m}^{-1}$, vacuum permittivity, $\left(1 / 4 \pi \epsilon_{0}\right)=8.987 \cdot 10^{9} \mathrm{~F}^{-1} \mathrm{~m}$;

$c=2.997 \cdot 10^{8} \mathrm{~m} \cdot \mathrm{s}^{-1}$, velocity of light;

$m_{\mathrm{p}}=1.672 \cdot 10^{-27} \mathrm{~kg}$, proton mass.

\subsection{Symbols used for the ion probe beam}

Pencil-beam diameter: $\phi_{\mathrm{i}}$, intensity: $I_{\mathrm{i}}$.

$A, Z$ atomic mass and charge, $m_{\mathrm{i}}=A \cdot m_{\mathrm{p}}, Q=Z \cdot q,(Z=1$ in the present case $)$.

We mainly used argon $(A=40)$ or xenon $(A=129)$.

Nominal velocity $v_{0}\left[\mathrm{~m} \cdot \mathrm{s}^{-1}\right]$.

The ion gun is at potential $U_{\mathrm{s}}$ such that the nominal kinetic energy $E_{\mathrm{k}}=(1 / 2) m_{\mathrm{i}} v_{0}^{2}=$ $Q U_{\mathrm{s}}$; for argon $v_{0}\left(E_{\mathrm{k}}=2 \mathrm{keV}\right)=9.8 \cdot 10^{4} \mathrm{~m} \cdot \mathrm{s}^{-1}, v_{0}\left(E_{\mathrm{k}}=5 \mathrm{keV}\right)=1.55 \cdot 10^{5} \mathrm{~m} \cdot \mathrm{s}^{-1}$.

\subsection{Symbols used for the proton beam $(A=1, Z=1)$}

$\beta_{0}=v_{\text {proton }} / c$

r.m.s. bunch length: $\Delta_{\mathrm{s}}[\mathrm{m}], L_{\mathrm{b}} \equiv \Delta_{\mathrm{s}} \cdot \sqrt{2 \pi}, \sigma_{\mathrm{t}} \equiv L_{\mathrm{b}} / \beta_{0} c[\mathrm{~s}]$,

r.m.s. transverse dimensions: $\sigma_{x}$ and $\sigma_{y}[\mathrm{~m}], \Delta_{\mathrm{r}}=\left[\sigma_{x}^{2}+\sigma_{y}^{2}\right]^{1 / 2}$.

For round beams $\sigma_{x}=\sigma_{y}=\sigma \Rightarrow \Delta_{\mathrm{r}}=\sqrt{2} \cdot \sigma$,

$n_{\mathrm{b}}$ : number of protons per bunch,

$V_{0} \equiv\left(q / 4 \pi \epsilon_{0}\right)\left(2 n_{\mathrm{b}} / L_{\mathrm{b}}\right)[\mathrm{V}]$,

$T[\mathrm{~s}]$ time interval between bunches.

\subsection{SPS machine parameters}

The SPS operated in fixed-target mode with the following parameters:

- total number of circulating protons: $1.8 \cdot 10^{13}-2.4 \cdot 10^{13}$,

- number of bunches: 4200, 
$-n_{\mathrm{b}}=4.3 \cdot 10^{9}-5.7 \cdot 10^{9} ; \sigma_{\mathrm{t}}=0.5 \mathrm{~ns} ; T \cong 5 \mathrm{~ns}$

- revolution frequency $44 \mathrm{kHz}$,

- momentum versus time $p(t)=14+0.1253(t-1250)[\mathrm{GeV} / c]$ where $t[\mathrm{~ms}]$ refers to the first injection,

- the monitor is installed at a location close to a wire-scanner where $\beta_{\mathrm{h}}=94.61 \mathrm{~m}$, $\beta_{\mathrm{v}}=22 \mathrm{~m}$ are the betatron functions, and $D_{\mathrm{h}}=2.83 \mathrm{~m}$ the dispersion.

\subsection{Important remarks}

In the present paper the analysed beam will consist of protons. This is not essential but will simplify the reading of the paper since any mention of ions will refer to the probe beam while any mention of protons will refer to the probed beam.

All the simulations, are made for a proton beam whose horizontal r.m.s. dimension and vertical r.m.s. dimension are equal to $\Delta_{\mathrm{r}}=1 \mathrm{~mm}$, and for a distance between the proton beam and the collector of $L=0.4 \mathrm{~m}$.

\section{PRINCIPLE}

The system of coordinates is defined in Fig. 1(a) whilst the detector principle is described in Fig. 1(b).
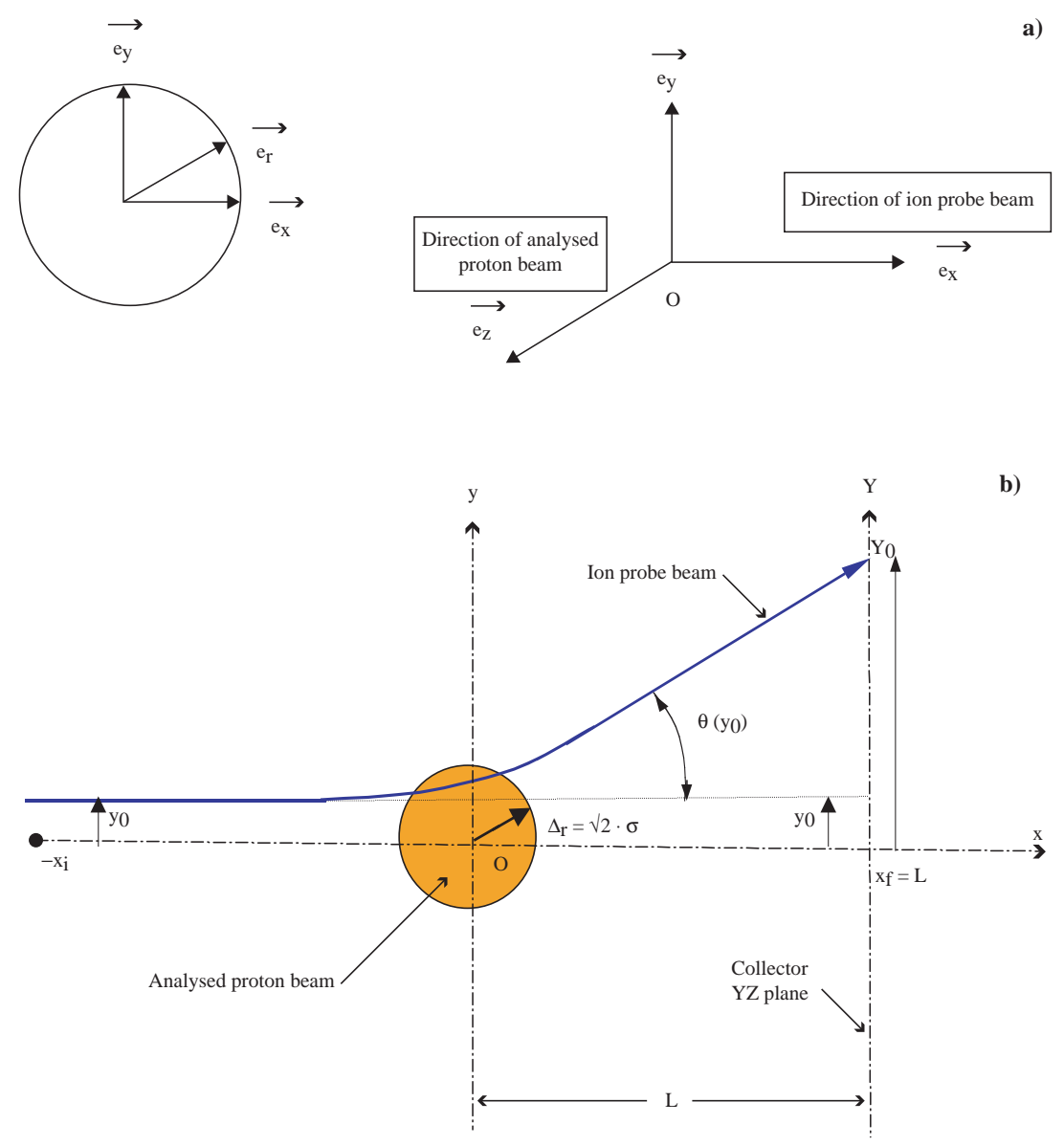

Fig. 1: (a) System of coordinates. (b) Symbols used and principle of the ion pencil-beam 'profilometer'. 
A low-energy $\left(U_{\mathrm{s}}=2-5 \mathrm{kV}\right)$ ion beam is generated at: $\left(x=-x_{\mathrm{i}}, y=y_{0} \ll\left|x_{\mathrm{i}}\right|, z=\right.$ 0 ) and moves with initial velocity $\vec{v}_{\mathrm{i}}=v_{0} \vec{e}_{x}$ perpendicular to the direction of the proton beam. The proton beam itself, centred at $(0,0,0)$ moves with velocity $\vec{v}_{\text {proton }}=\beta_{0} c \vec{e}_{z}$.

The proton beam is supposed to be very dense and has a transverse distribution function with r.m.s. values $\sigma_{x}$ and $\sigma_{y}$ and a uniform longitudinal distribution over $L_{\mathrm{b}}$. For simplicity we suppose a round Gaussian beam so that the normalized transverse distribution is given by:

$$
n_{\perp}(r)=\frac{2}{2 \pi \Delta_{\mathrm{r}}^{2}} \exp \left(-\frac{r^{2}}{\Delta_{\mathrm{r}}^{2}}\right), \quad r=\left(x^{2}+y^{2}\right)^{1 / 2} .
$$

The restriction to a Gaussian distribution and to a round beam is not essential; in Ref. [2] it is clearly demonstrated that the statements hereafter still remain valid for other realistic proton distributions. This has also been shown in later papers (Refs. [1b] and $[1 \mathrm{c}])$.

The protons are supposed to be relativistic $\left(\beta_{0} \cong 1\right)$ so that the electric spacecharge field of the proton bunch is mainly radial $\vec{E}_{\mathrm{r}}=E_{\mathrm{r}} \vec{e}_{\mathrm{r}}$. The relativistic condition is not absolutely necessary [2] but will be considered as being fulfilled in this paper except where mentioned.

On its way from $-x_{\mathrm{i}}$ to $x_{\mathrm{f}}$, where the collector is placed, an ion will be deflected by an angle $\theta$ as a result of the transverse proton space-charge electric field $\vec{E}_{\mathrm{r}}$. For each 'impact parameter' $y_{0}$ the ion collector in the observer plane $\left(x=x_{\mathrm{f}}=L\right.$, defined by $\vec{e}_{y}$ and $\vec{e}_{z}$ ) will record a spot at $Y=Y_{0}=y_{0}+L \theta\left(y_{0}\right)$, when the proton beam is ON, and at $Y=y_{0}$ when the proton beam is OFF. Then the deflection angle $\theta\left(y_{0}\right)$ can be obtained by:

$$
\operatorname{tg} \theta\left(y_{0}\right) \cong \theta\left(y_{0}\right)=\frac{Y_{0}-y_{0}}{L}
$$

\subsection{Case 1}

We suppose here that the ion velocity $v_{0}$ and the bunch length $L_{\mathrm{b}}$ are such that the ion interacts only once with the bunch on its way from the gun to the detector. Considering that the proton beam electric field effective range is $\pm 5 \cdot \Delta_{\mathrm{r}}$ around the $\mathrm{Oz}$ axis, this condition can be expressed as follows:

$$
\frac{10 \cdot \Delta_{\mathrm{r}}}{v_{0}} \leq \sigma_{\mathrm{t}}, \quad(\text { condition } 1)
$$

Condition 1 is of course fulfilled for unbunched proton beams.

It has been shown ([1], [2] and Appendix A) that, in the case of a Gaussian beam, with minor simplifications the deviation angle $\theta$ can be expressed by:

$$
\theta\left(y_{0}\right)=\frac{Q V_{0}}{2 E} \pi \cdot \operatorname{erf}\left(\frac{y_{0}}{\Delta_{\mathrm{r}}}\right)=\theta_{\max } \operatorname{erf}\left(\frac{y_{0}}{\Delta_{\mathrm{r}}}\right)
$$

with

$$
\theta_{\max } \equiv \frac{Q V_{0}}{2 E_{\mathrm{k}}} \pi[\mathrm{rad}]
$$


and

$$
\text { the error function } \quad \operatorname{erf}(u) \equiv \frac{2}{\sqrt{\pi}} \int_{0}^{u} e^{-\xi^{2}} \mathrm{~d} \xi,
$$

some properties of the error function are:

$$
\operatorname{erf}(\infty)=1, \operatorname{erf}(0)=0, \operatorname{erf}(x)=-\operatorname{erf}(-x)
$$

In the case of an unbunched beam the parameter $V_{0} \equiv\left(q / 4 \pi \epsilon_{0}\right)\left(2 n_{\mathrm{b}} / L_{\mathrm{b}}\right)[\mathrm{V}]$, used for $\theta_{\max }$, has to be revised, namely, $n_{\mathrm{b}}$ has to be replaced by the number of circulating protons and $L_{\mathrm{b}}$ by the machine circumference.

By differentiation of Eq. (2a)

$$
\frac{\mathrm{d} \theta\left(y_{0}\right)}{\mathrm{d} y_{0}}=\frac{2 \theta_{\max }}{\sqrt{\pi} \Delta_{\mathrm{r}}} \exp \left(-\frac{y_{0}^{2}}{\Delta_{\mathrm{r}}^{2}}\right),
$$

and so the proton beam transverse r.m.s. dimension

$$
\Delta_{\mathrm{r}}=\frac{2 \theta_{\max }}{\sqrt{\pi}} \frac{1}{\left.\frac{\mathrm{d} \theta}{\mathrm{d} y_{0}}\right|_{y_{0}} \cong 0}
$$

is evaluated.

\subsection{Case 2}

In the case where condition 1 is not fulfilled, i.e. $\left(10 \cdot \Delta_{\mathrm{r}} / v_{0}\right) \gg \sigma_{\mathrm{t}}$, the ion will receive small kicks at each passage of a bunch, of duration $\sigma_{\mathrm{t}}$, and will not be subject to an electric force (straight line trajectory) during the time interval between two bunches which is about $T-\sigma_{\mathrm{t}}$. The trajectory is more complicated. A rough approximation can, however, be obtained by simply replacing in Eqs. (2a) and (2b)

$$
V_{0} \equiv \frac{q}{4 \pi \epsilon_{0}} \frac{2 n_{\mathrm{b}}}{L_{\mathrm{b}}}
$$

by

$$
V_{0}^{\prime} \equiv \frac{q}{4 \pi \epsilon_{0}} \frac{2 n_{\mathrm{b}}}{L_{\mathrm{b}}} \cdot \frac{\sigma_{\mathrm{t}}}{T}
$$

Numerical values: With the data taken from the SPS (Section 2.4 ), and $E_{\mathrm{k}}=2 \mathrm{keV}$, we obtain $V_{0}=173 \mathrm{~V}, \theta_{\max }=75 \mathrm{mrad}$ [from Eq. (2b)] and $\theta_{\max } \frac{\sigma_{t}}{T}=7.5 \mathrm{mrad}$.

Figure 2(a) represents a simulation of $\theta(y)$, Eq. (2a), and of its derivative equivalent to Eq. (2d). On Fig. 2(b) we represent the influence of $\Delta_{\mathrm{r}}$ and that of the proton beam position on the deflection angle measurement. Once Eq. (2d) is evaluated, a normalization is applied, namely

$$
\left(\frac{1}{2 \theta_{\max }}\right) \cdot \frac{\mathrm{d} \theta\left(y_{0}\right)}{\mathrm{d} y_{0}}=\frac{1}{\sqrt{\pi} \Delta_{\mathrm{r}}} \exp \left(-\frac{y_{0}^{2}}{\Delta_{\mathrm{r}}^{2}}\right)
$$

which is equivalent to Eq. (1) and therefore represents the distribution that we intend to retrieve from our measurements. It is then straightforward to retrieve $\Delta_{\mathrm{r}}$ from Eq. (2e). From the curves one can observe that $\theta(y)$ is about linear around $y=0$ and that $\theta(|y| \geq$ $\left.\Delta_{\mathrm{r}}\right) \cong \theta_{\max }$. This asymptotic behaviour is an important characteristic of the detector since 
it implies that the deflecting angle reaches its maximum when the impact parameter is about equal to the proton-beam transverse r.m.s. dimension.
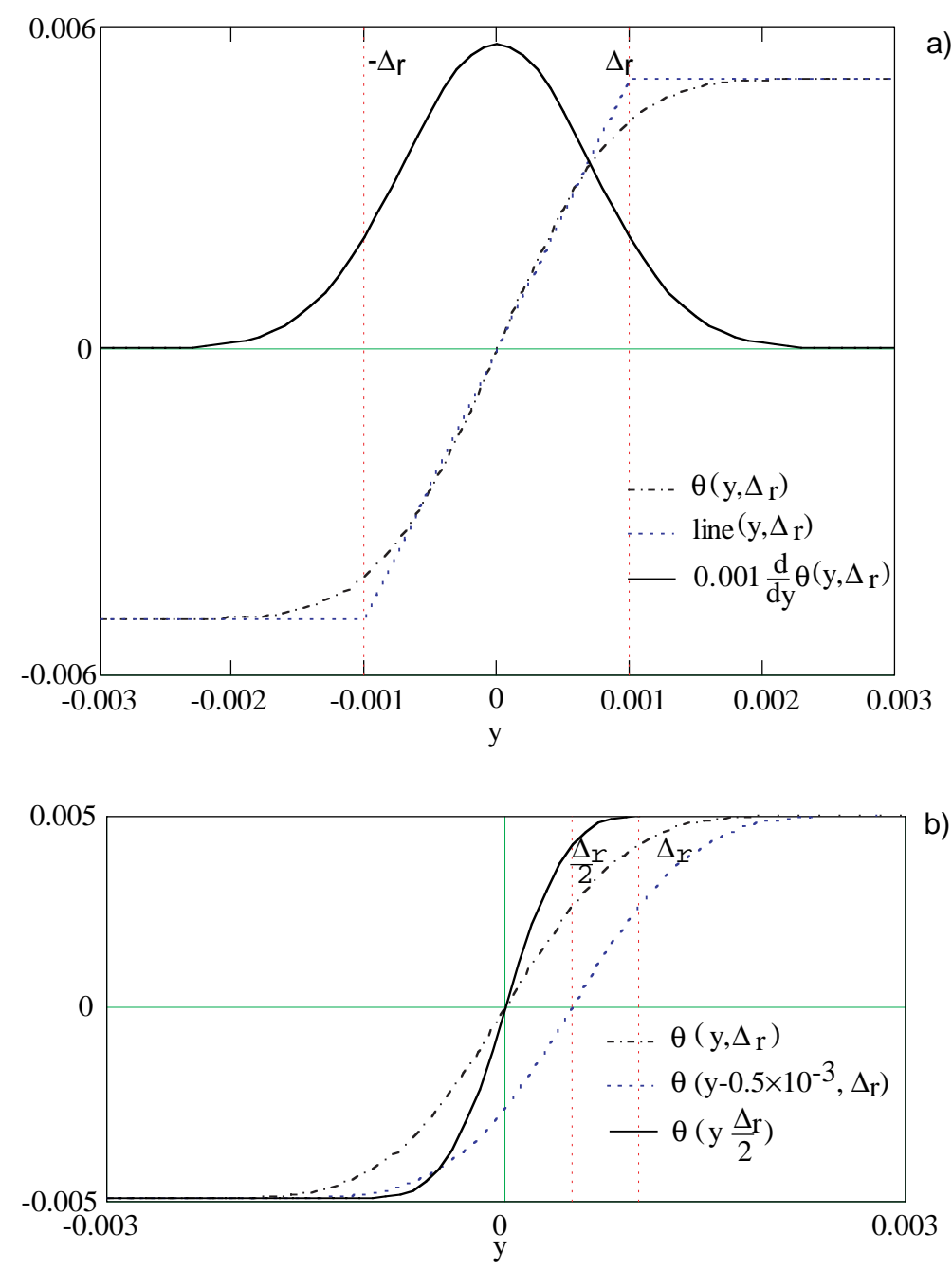

Fig. 2: Simulations of the deflection angle. (a) Plot of the deflection angle $\theta(y)=\theta_{\max } \operatorname{erf}\left(y / \Delta_{\mathrm{r}}\right)$ [Eq. (2a)] for $\Delta_{\mathrm{r}}=10^{-3} \mathrm{~m}$ and $\theta_{\max }=5 \cdot 10^{-3} \mathrm{rad}$. The dotted curve named 'line $\left(y, \Delta_{\mathrm{r}}\right)$ ' is a linearization of $\theta(y)$ by straight lines; it appears that the maximum deflection angle occurs for $y \cong \Delta_{\mathrm{r}}$. A plot of $\mathrm{d} \theta(y) / \mathrm{d} y$, multiplied by an arbitrary factor is also given so as to show that the initial Gaussian distribution [equivalent to Eq. (1)] is retrieved in such a way. (b) Plot of $\theta\left(y, \Delta_{\mathrm{r}}\right), \theta\left(y, \Delta_{\mathrm{r}} / 2\right), \theta\left(y-5 \cdot 10^{-4} \mathrm{~m}, \Delta_{\mathrm{r}}\right)$ to illustrate the influence of the proton-beam r.m.s. dimension and position on the deflection angle measurement.

\section{$\underline{\text { Remarks }}$}

- In practice, instead of a single ion, one uses an ion pencil-beam of small diameter $\phi_{\mathrm{i}}$ and small intensity $I_{\mathrm{i}}$. The collector is aimed then to measure the centre of gravity of the impinging pencil beam.

- For a given ion kinetic energy $E_{\mathrm{k}}$ and ion charge, the maximum deviation angle given by Eq. ( $2 \mathrm{~b})$ is independent of the ion mass. The angle $\theta_{\max }$ is proportional to the number of protons per bunch $n_{\mathrm{b}}$. 
- As complementary information, this monitor will also provide the proton-beam position $y_{\mathrm{cg}}$ where the ion is not deflected $\left[\theta\left(y_{\mathrm{cg}}\right)=0 \mathrm{rad}\right]$.

- In the case where condition 1 is not fulfilled, a modulation of the deflection angle with time occurs [2] since the ion will sense no electric field during the time interval between two consecutive bunches. This effect is quite small and even negligible in the present case where we use a heavy probe ion $\left(\mathrm{Ag}^{+}\right.$or $\left.\mathrm{Xe}^{+}\right)$moving at relatively small velocity. A detailed simulation program has been developed [2]. It includes all the beam parameters, namely the proton-beam longitudinal and transverse distribution and the deviation angle modulation effect due to the proton-beam bunching factor. This simulation code, which also includes the detector environment (such as the vacuum pipe dimensions, etc.), is a good tool for interpreting and analysing our experimental results.

\subsection{More on data treatment}

Having measured $\theta\left(y_{0}\right)$, where $y_{0}$ takes its values over a significant interval around the proton beam (at least $-\Delta_{\mathrm{r}} \leq y_{0} \leq \Delta_{\mathrm{r}}$ ), one can deduce $\theta_{\max }$ and $\mathrm{d} \theta\left(y_{0}\right) / \mathrm{d} y_{0}$ [Eq. (2d)]. The latter equation [equivalent to Eq. (1)] represents the density distribution in the proton bunch in the $y$ direction. The radial r.m.s. value $\Delta_{\mathrm{r}}$ can then be obtained from Eq. (2e). However, in some cases this method of data treatment might not be very precise, because some errors are induced by the numerical derivation of $\theta\left(y_{0}\right)$. It is then employed only for rough estimates, and more elaborate numerical smoothing techniques are needed.

In some cases, considering a Gaussian beam distribution, after having measured the deviation angle $\theta$ as a function of $y_{0}$ (or $y$ ), we proceed with the 'error-function fit' (erf fit) on the measured data. In other words, we make use of an expression like Eq. (2a) which almost fits the measured data. An error function is available on most processors. The fit gives the most probable value of $\Delta_{\mathrm{r}}$. One then computes $\partial \theta_{\text {fit }} / \partial y_{0}$ so as to trace the bunch distribution in the $y$ direction.

\section{THE DIFFERENT COLLECTOR TYPES}

The aim of the collector is to measure the impinging ion-beam position (or more precisely its centre of gravity), equal to $y_{0}$ when the proton beam is OFF, and to $Y_{0}$ when the beam is ON.

The ion current intensity is rather small (a fraction of $n A$ ) such that in some cases the use of Micro-Channel Plates (MCPs), which convert the incoming ions into electrons, might become necessary. The MCP gain can be of the order of $10^{4}$.

We have used three different position detectors with and without MCPs:

a) Equally spaced metallic microstrips. Up to 32 strips, spaced at $1.5 \mathrm{~mm}$, were used. The current collected by each strip is integrated before being processed. The scanning time through the proton beam can be as low as $1 \mathrm{~ms}$.

b) A resistive plate with adequate time constant. An integrator is placed at each end, $A$ and $B$, of the plate. The ratio $D / S=(A-B) /(A+B)$ is proportional to the centre of charges (or centre of gravity) of the impinging beam. The overall scanning time can be of the order of $0.1 \mathrm{~ms}$.

c) A luminescent screen viewed by a CCD camera. The image is processed every 20 (or 40) ms so as to determine the ion-beam position (or more precisely its centre of 
gravity) or, as we shall see in Section 9, the ion density distribution. There is also the possibility of sampling the probe beam within a very short time (about $1 \mu \mathrm{s}$ ); in this case the measurement duration is $1 \mu \mathrm{s}$, though the image processing time can be much longer.

In the case of a stored or stable proton beam it is easy to proceed with averaging and/or filtering. Therefore, in the case of a stored beam, at fixed energy and intensity, one can also foresee the monitor being as a 'watch-dog' continuously measuring the maximum deviation angle; in other words, the variation of the transverse r.m.s. proton beam dimension with time.

\section{ION SOURCE MAIN PARAMETERS}

The ion source (gun + associated optics) plays an important role; and is an industrial product with significant modifications implemented by us.

The source can provide not only a pencil beam but can also be arranged so as to deliver an ion curtain or a wide cylindrical beam. In this Section we shall nevertheless limit our analysis to the pencil-beam source.

It is evident that the source must be a) as intense as possible in order to obtain good accuracy from the collector; b) have small diameter $\phi_{\mathrm{i}}$, a fraction of $\Delta_{\mathrm{r}}$; and c) have a small divergence, a fraction of $\theta_{\max }$.

Measurements showed [2] that, $15 \mathrm{~mm}$ after the gun output, the emittance of a $E_{\mathrm{k}}$ $=2 \mathrm{keV}$ ion beam is $5.5 \mathrm{~mm} \cdot \mathrm{mrad}$ for an ion current $I_{\mathrm{i}}=50 \mathrm{nA}$ (and $4 \mathrm{~mm} \cdot \mathrm{mrad}$ when $\left.E_{\mathrm{k}}=5 \mathrm{keV}\right)$.

After the ion gun (at potential $U_{\mathrm{s}}$ such that $E_{\mathrm{k}}=Q \cdot U_{\mathrm{s}}$ ) the ion beam passes through a drift tube. At the drift tube output the ion beam is focused by an 'Einzel lens' and then collimated. The collimator system consists of slits restricting the beam dimension and angle in the plane of interest. At the collimator output the beam is deflected by electrically charged plates so that it crosses the proton beam at an angle. A future version of the ion source foresees a parallel displacement to vary the impact parameter $y_{0}$.

We require a beam with diameter $\phi_{\mathrm{i}}=0.2 \mathrm{~mm}$ and divergence $1.0 \mathrm{mrad}$ in the plane of interest. In such a case the current is reduced down to about $1 \mathrm{nA}$ by the slits acting as collimators. Such a current, collected on one strip of the detector, will result in a voltage of $20 \mathrm{mV}$ when integrated during $1 \mathrm{~ms}$ by a $50 \mathrm{pF}$ capacitance. Such a signal can be accurately processed. More collimated beams and therefore more reduced intensities require the use of an MCP acting as amplifier.

On its way from the source to the collector, at distance $L_{x}$, the ion pencil-beam of radius $r_{\mathrm{i}}=\phi_{\mathrm{i}} / 2$ will naturally expand as a consequence of its own transverse electric field. For a uniformly distributed round ion beam the increase in radius is about

$$
\Delta_{\mathrm{r}_{\mathrm{i}}}=\frac{Q}{m_{\mathrm{i}}} \frac{1}{4 \pi \epsilon_{0}} \frac{I_{\mathrm{i}}}{v_{0}^{3}} \frac{L_{x}^{2}}{r_{\mathrm{i}}}
$$

which for argon, $I_{\mathrm{i}}=1 \mathrm{nA}, r_{\mathrm{i}}=0.1 \mathrm{~mm}, E_{\mathrm{k}}=2 \mathrm{keV}, L_{x}=1 \mathrm{~m}$, gives $\Delta_{\mathrm{r}_{\mathrm{i}}}=0.23 \mathrm{~mm}$, which is just acceptable. In practice $\Delta_{\mathrm{r}_{\mathrm{i}}}$ is smaller since the ion beam is collimated in one direction only, namely along $\vec{e}_{y}$ and is rather wide along the $\vec{e}_{z}$ direction. In the case of such a rectangular-shaped beam of height $\phi_{\mathrm{i}}$ and width $d_{\mathrm{i}} \cong 1 \mathrm{~cm}, r_{\mathrm{i}}$ must be replaced by $d_{\mathrm{i}}$ in Eq. (3). The space-charge potential $\Delta U_{\mathrm{s}}$ between the centre and the edge of the ion beam 
will induce a corresponding difference in kinetic energy and therefore different deflection angles. Since for a round beam $\Delta U_{\mathrm{s}}=30 \cdot I_{\mathrm{i}} \cdot c / v_{0}$, the change of deflection angle can be expressed as follows:

$$
\left|\frac{\Delta \theta_{\max }}{\theta_{\max }}\right|=\frac{\Delta E_{\mathrm{k}}}{E_{\mathrm{k}}}=\frac{\Delta U_{\mathrm{s}}}{U_{\mathrm{s}}}=\frac{30 \cdot I_{\mathrm{i}} \cdot c}{v_{0} \cdot U_{\mathrm{s}}} .
$$

For $U_{\mathrm{s}}=2 \mathrm{kV}$ and $I_{\mathrm{i}}=1 \mathrm{nA}$ this ratio is very small.

It is evident that higher intensity and lower emittance sources are welcome and under investigation.

\section{CHOICE OF THE PROBE PARTICLE TYPE}

Up to now we have considered heavy ions to be used as probe particles. One might of course consider instead the use of an electron pencil-beam (velocity: $v_{\mathrm{e}}$, mass: $m_{\mathrm{e}}$ ) whose production is easier. In such a case the effect of the proton-beam azimuthal magnetic field $\vec{B}_{\varphi}$ (with $\left|\vec{B}_{\varphi}\right|=\frac{\beta_{0}}{c}\left|E_{\mathrm{r}}\right|$ ) has to be taken into account.

For an ion with velocity $v_{0}$ the ratio of the electric force $F_{\mathrm{e}}(r)$ to the magnetic force $F_{\mathrm{b}}(r)$, at a given radius $r$, is:

$$
\left|\frac{F_{\mathrm{e}}(r)}{F_{\mathrm{b}}(r)}\right|=\frac{1}{\beta} \frac{c}{v_{0}} .
$$

Considering the same maximum deviation angle $\theta_{\max }$, for both ion and electron beams, the electron velocity will be $v_{\mathrm{e}}=v_{0} \cdot\left(A \cdot m_{\mathrm{p}} / m_{\mathrm{e}}\right)^{1 / 2} \gg v_{0}$ and so:

$$
\left|\frac{F_{\mathrm{e}}(r)}{F_{\mathrm{b}}(r)}\right|_{\text {electrons }} \leq \frac{1}{44 \cdot \sqrt{A}}\left|\frac{F_{\mathrm{e}}(r)}{F_{\mathrm{b}}(r)}\right|_{\text {ion }} .
$$

Compared with those of ions, the electron trajectories are significantly influenced by the proton beam self-magnetic field. In other words, the electron global trajectory is not straightforward and the measurement of $\theta\left(y_{0}\right)$ is rather difficult. Examples are given in Ref. [2].

Furthermore, the deflection angle modulation, resulting from the ratio $\sigma_{\mathrm{t}} / T<1$ will be enhanced due to the higher velocity of the electron. Indeed the electron will move over a longer distance between two bunches where it is subject to no transverse electric field.

This is why a heavy-ion source (up to xenon) has been used instead of an electron gun.

\section{PROFILE MEASUREMENT USING AN ION PENCIL-BEAM}

\subsection{Principle}

The detector is essentially based on the principle explained in Section 3. A detailed description of the detector and of the measurements on the CERN SPS machine are given in Ref. [2] from which we extract the essential parts.

The principle is shown in Fig. 3(a) and is self-explanatory. It must be noticed, however, that in order to simplify the hardware, the ion beam does not cross the proton beam perpendicularly $\left[\vec{v}_{\mathrm{i}}=v_{\mathrm{i} x} \vec{e}_{x}+v_{\mathrm{i} y} \vec{e}_{y}\right.$, with $v_{\mathrm{i} y} \neq 0$, see Fig. 1(a)] but at an angle $\varphi$ determined by the deflecting plates. This is illustrated by Fig. 3(b). In the future we shall 


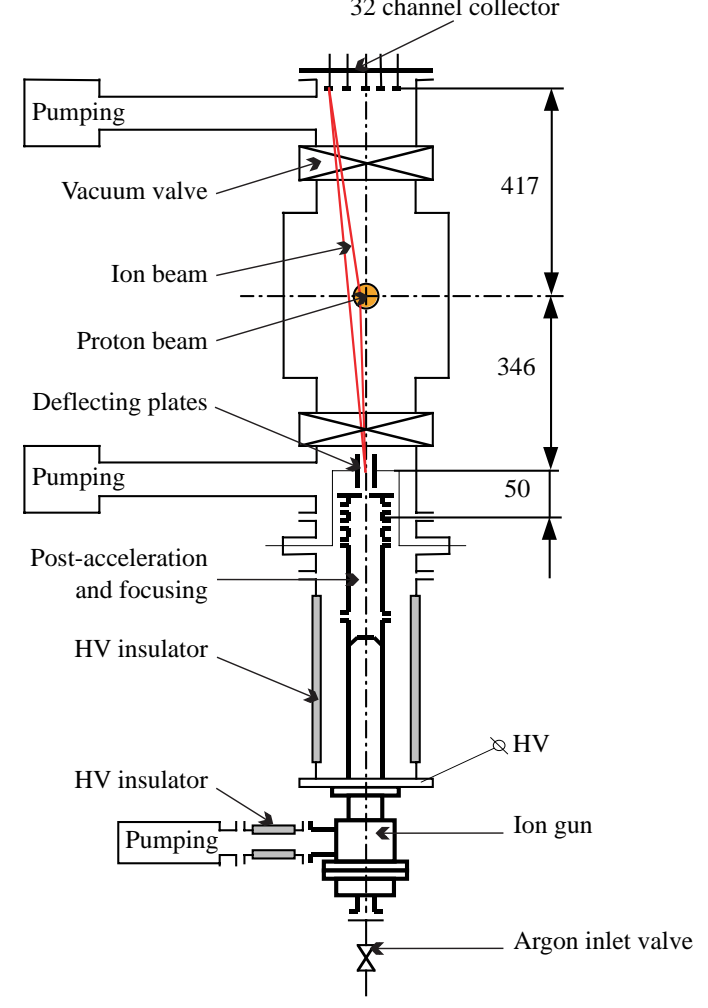

a)

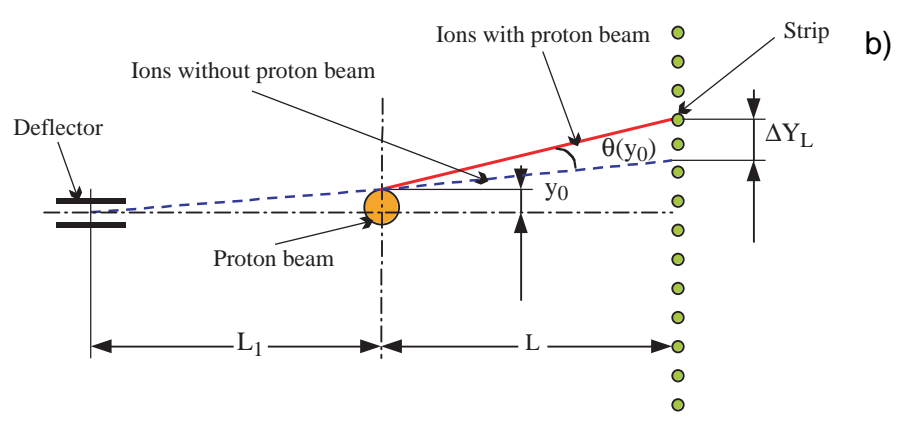

Fig. 3: Ion pencil-beam technique. (a) Experimental set-up as implemented on the SPS machine. The ion beam coming out of the gun is post-accelerated and focused by an 'Einzel lens'. The ion, electrically deviated by plates, passes through the probed proton beam, where it is deflected and finally reaches the collector. All dimensions are in millimetres. (b) Details of 3(a) where it is shown that in the present set-up the ion beam is not perpendicular to the proton beam but has a small incident angle.

improve our optical system in order to render the ion beam completely parallel to $\left(x_{\mathrm{i}}, x_{\mathrm{f}}\right)$ and therefore reduce the velocity component $v_{\mathrm{i} y}$ as much as possible. Nevertheless, since in our experiment $L_{1}$ and $L$ are much larger than the analysed beam size, this effect is negligible.

The collector consists of 32 strips of $1 \mathrm{~mm}$ width, spaced at $1.5 \mathrm{~mm}$. The detector can be isolated completely from the machine by two vacuum valves. Vacuum pumps ensure that practically no pressure increment is recorded on the machine. 


\subsection{Determination of the deflection angle}

The computation will depend on whether the ion pencil-beam has a velocity component $v_{\text {iy }}$ equal to zero or not.

7.2.1 The ion beam has no component $v_{\mathrm{i} y}$ prior to its interaction with the proton beam In such a case $\vec{v}_{\mathrm{i}}=v_{\mathrm{i} x} \vec{e}_{x}$. The ordinate $Y$ is determined by:

$$
Y=y+L \cdot \theta(y) \equiv g(y) \text { or } y=g^{-1}(Y)
$$

and

$$
\frac{\mathrm{d} Y(y)}{\mathrm{d} y}=1+L \cdot \frac{\mathrm{d} \theta(y)}{\mathrm{d} y}=\frac{\mathrm{d} g(y)}{\mathrm{d} y}, \quad \text { or } \quad \frac{\mathrm{d} y(Y)}{\mathrm{d} Y}=\frac{\mathrm{d}\left(g^{-1}(Y)\right)}{\mathrm{d} Y}=\frac{1}{g^{\prime}(Y)} .
$$

7.2.2 The ion beam has a component $v_{\mathrm{i} y}$ prior to its interaction with the proton beam

In the case of a beam having a velocity component along $\vec{e}_{y}$ i.e $\vec{v}_{\mathrm{i}}=v_{\mathrm{i} x} \vec{e}_{x}+$ $v_{\text {i } y} \vec{e}_{y}$, (with $\left.v_{\text {i } y} \ll v_{\text {ix }}\right)$, we set $\varphi(y) \cong \operatorname{tg}(\varphi(y))=v_{\text {iy }}(y) / v_{\text {ix }}(y)$. Then

$$
Y=y+L \cdot(\theta(y)+\varphi(y)) \equiv g_{1}(y) \quad \text { or } \quad y=g_{1}^{-1}(Y)
$$

and

$$
\frac{\mathrm{d} Y(y)}{\mathrm{d} y}=1+L \cdot\left(\frac{\mathrm{d} \theta(y)}{\mathrm{d} y}+\frac{\mathrm{d} \phi(y)}{\mathrm{d} y}\right)=\frac{\mathrm{d} g_{1}(y)}{\mathrm{d} y}, \quad \text { or } \quad \frac{\mathrm{d} y(Y)}{\mathrm{d} Y}=\frac{\mathrm{d}\left(g_{1}^{-1}(Y)\right.}{\mathrm{d} Y}=\frac{1}{g_{1}^{\prime}(Y)} .
$$

The ordinate $Y$ is determined, in practice, by measuring the deflected ion beam's centre of charge or centre of gravity. From this measurement we determine the deflection angle using Eq. (5) or Eq. (7):

$$
\theta(y)=\frac{Y-y}{L} \quad \text { or } \quad \theta(y)=\left(\frac{Y-y}{L}\right)-\varphi(y)
$$

where $y$ and $\varphi(y)$ are measured when the proton beam is OFF and $L$ is well determined [Fig. (3b)].

\subsection{Measurements made on the SPS machine}

The ion beam had a diameter of $\phi_{\mathrm{i}}=0.2 \mathrm{~mm}$ and an intensity of a few $\mathrm{nA}$. The beam was displaced by changing the voltage on the plates. Measurements were made in the horizontal plane.

A first measurement aimed to prove the validity of the principle. The impact parameter $y_{0}$ is scanned every SPS cycle and the pencil-beam signal is integrated over 1 second. The measurement of the deflection angle $\theta$ as a function of $y_{0}$ is given by Fig. 4(a). The maximum deflection angle $\theta_{\max } \cong 12.5 \mathrm{mrad}$ is about that expected from numerical simulations since in the present case: $n_{\mathrm{b}}=9 \cdot 10^{9}$ (see subsection 3.2). Considering some fluctuation in the r.m.s. transverse dimension and in the position from cycle to cycle, the results are plausible. The integration time of $1 \mathrm{~s}$ averages out the proton r.m.s. dimension $\Delta_{\mathrm{r}}$ which varies during the SPS acceleration ramp. 

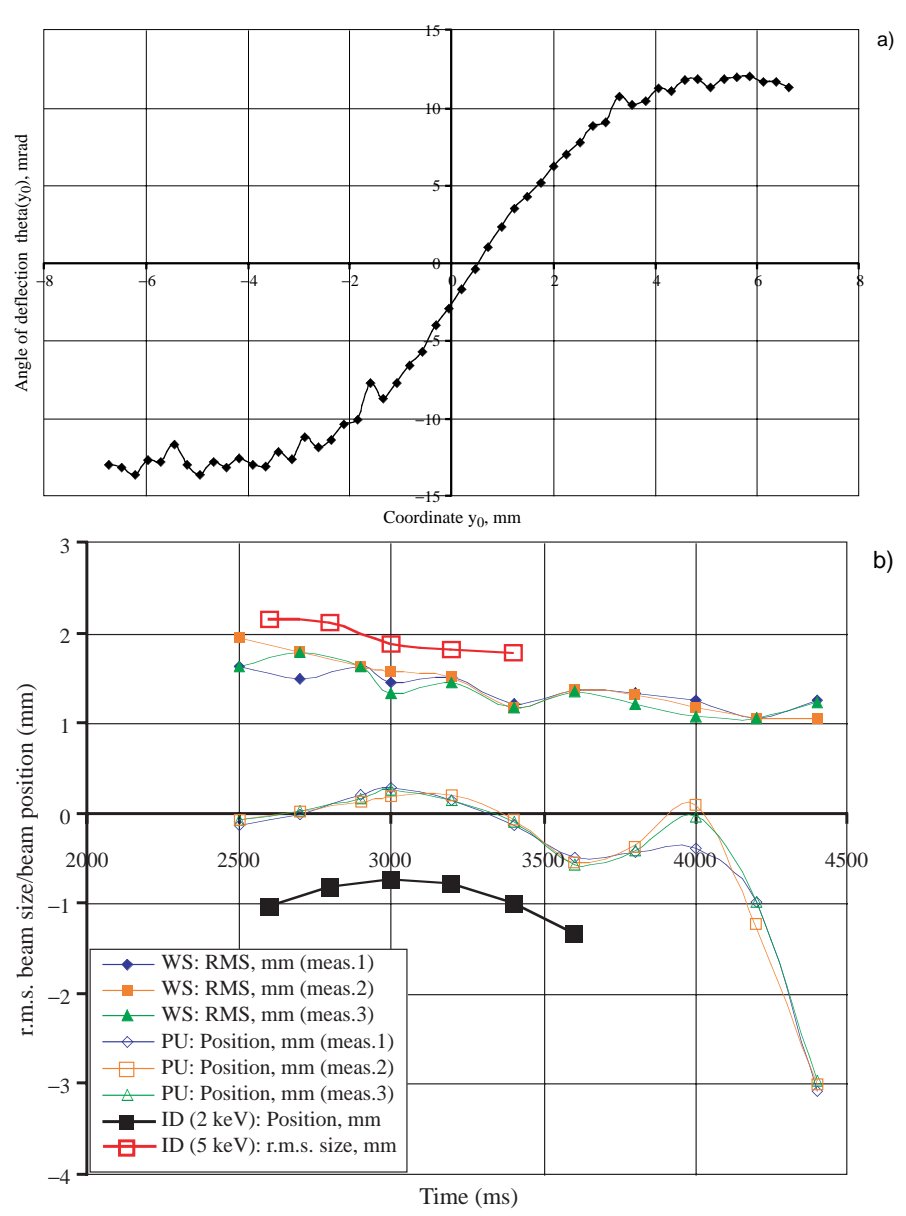

Fig. 4: Measurements made on the SPS in the horizontal plane, using the ion pencil-beam method. (a) Rough measurement of the deflection angle $\theta\left(y_{0}\right)$. The collector consists of metallic strips spaced at $1.5 \mathrm{~mm}$. (b) r.m.s. size (upper 4 curves) and position (lower 4 curves) measured along the SPS acceleration ramp with the ion profilometer (ID), the wire scanner (WS), and the beam-position pick-up (PU).

A second, more global measurement was intended to measure the r.m.s. size at different instants during the SPS acceleration cycle. The integration time was $200 \mathrm{~ms}$. The r.m.s. $\Delta_{\mathrm{r}}(t)$ was obtained by differentiating the angle of deviation $\theta\left(y_{0}\right)$ as given by Eq. (2e) (and therefore not applying an erf fit as explained in subsection 3.1). At the same instants we measured the proton-beam profile with a wire scanner, and its position with a standard pick-up. Three sets of measurements were made. The comparative results are shown in Fig. 4(b). The measurements are very similar. The r.m.s. beam size measured with the ion pencil-beam appears to be larger than that obtained with the wire scanner. As far as the proton-beam position is concerned, there is a good agreement between the measurements obtained with the ion pencil-beam and those made with the SPS pick-up. Studies are under way to explain the difference in the r.m.s. measurements. Anyway, we consider these preliminary experimental results to be promising. 
Instead of scanning the ion beam in the $y$ direction, we use a flat ion curtain beam, inclined by an angle $\varphi$. For this purpose the ion source has been modified. The principle becomes as described by Fig. 5(a). The thin ion beam is distributed along the line $y=z \cdot \operatorname{tg}(\varphi)$. When the proton beam is OFF, the corresponding line is directly recorded on the observer (or collector) plane orthogonal to the ion direction at $x=x_{\mathrm{f}}=L$. The geometry used between the ion source and the observer plane is explained in detail in Appendix B. A complete description of this type of monitor and of the measurements is given in Ref. [3].

When the proton beam in $\mathrm{ON}$, the ion ordinate $y$ is deflected by an angle $\theta(y)$ and reaches the observer plane at $Y=y+L \cdot \operatorname{tg} \theta(y) \cong y+L \theta(y)$.

After some linear transformations we retrieve the distribution $\theta(y)$ in a similar way to that explained in Section 3.

In the present case we used an MCP followed by a luminescent screen [see Section 4(c)]. An example of the observed 'curved' curtain beam is shown in Fig. 5(b). We applied an 'erf fit' to the measured deflection angle (see subsection 3.1). Measurements during the SPS cycle have been made and compared to those, made at the same instants, with the wire-scanner. Results are shown in Fig. 5(c) and demonstrate a good agreement between the measurements. The proton beam distributions obtained from the derivation of the fits are given in Fig. 5(d). The accuracy is limited by the image treatment [4], more precisely by the camera-limited number of pixels. The actual image processing also limits to $20 \mathrm{~ms}$ the time interval between two profile measurements.

Fig. 5: Ion curtain technique.

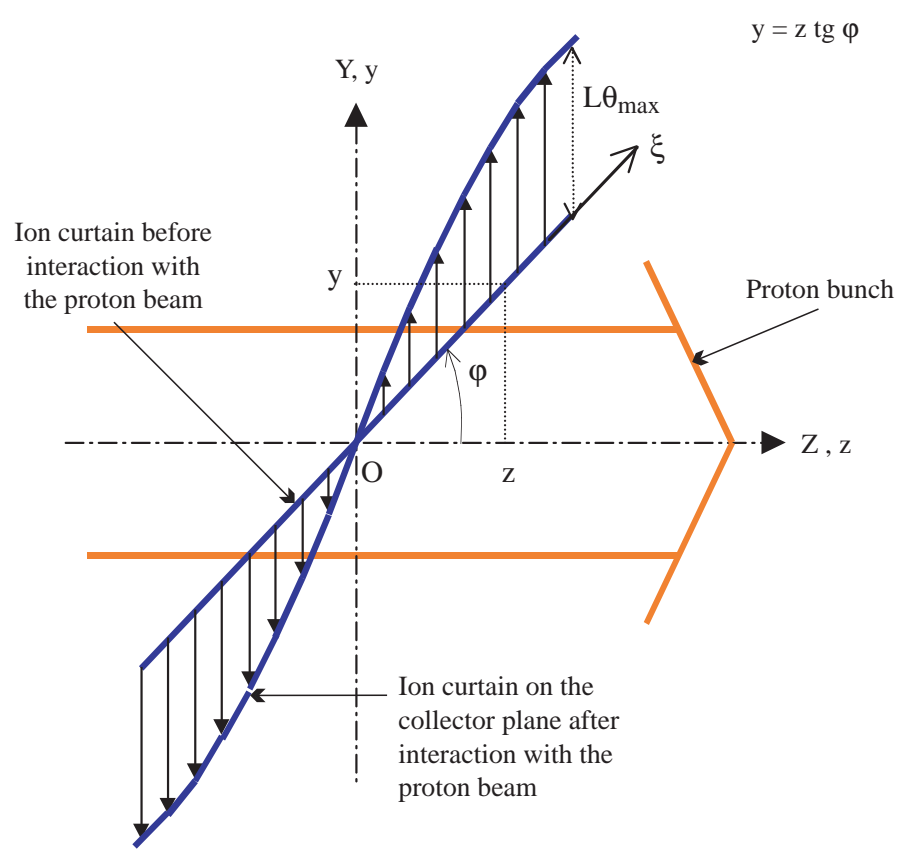

5(a) Principle of the ion curtain profilometer. Without proton beam the curtain lies on the line $0 \xi$ of equation $y=Y=Z \operatorname{tg}(\varphi)$ and moves perpendicular to the sheet. With proton beam, each individual ion at ordinate $y$ is deviated by angle $\theta(y)$ and reaches the collector at ordinate $Y=y+L \theta(y)$. 


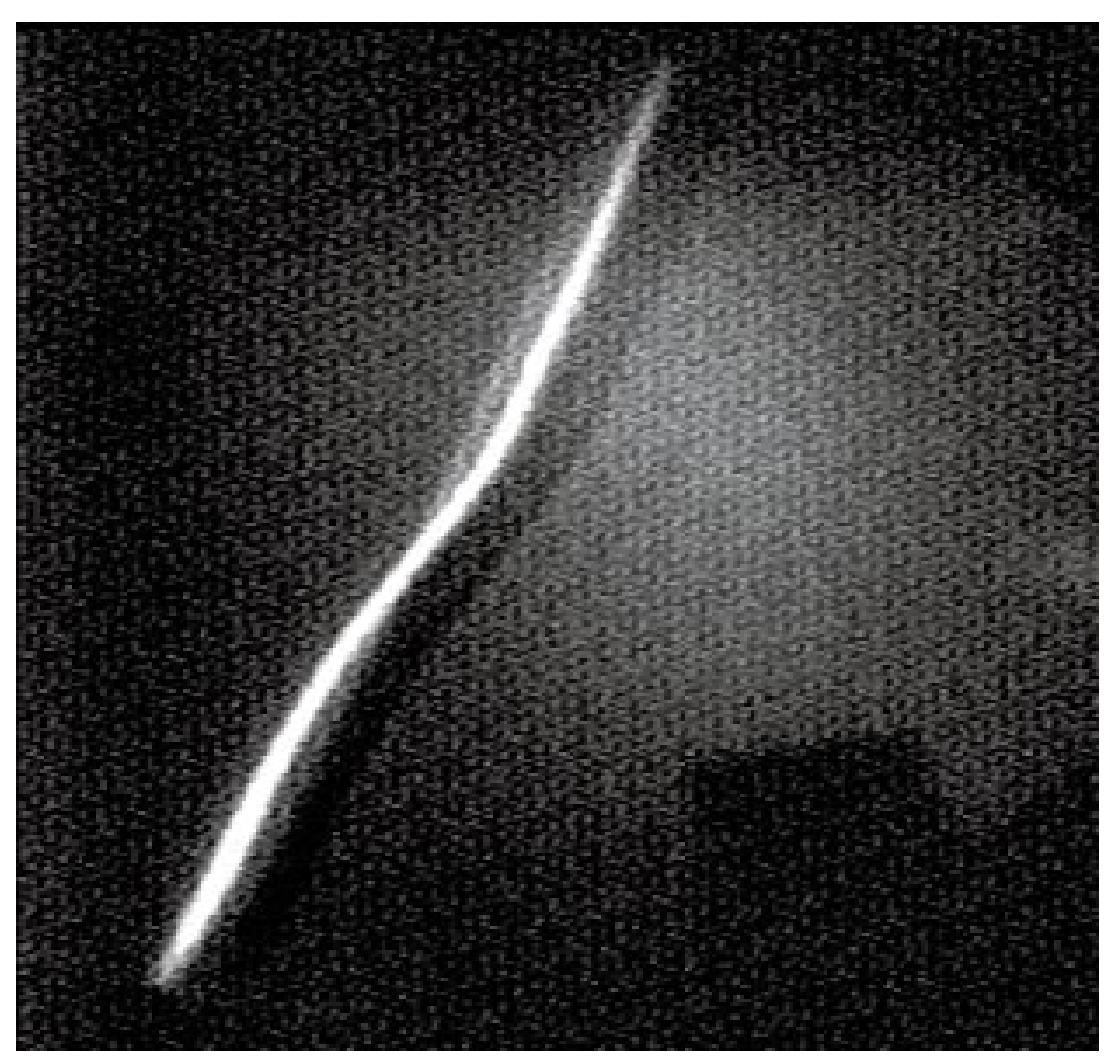

5(b) Image observed on a TV screen, showing the ion curtain twisted by the proton beam. The SPS momentum was $314 \mathrm{GeV} / c$ and the total number of circulating protons was $1.8 \cdot 10^{13}$. We used $\mathrm{Xe}^{+}$ions at $2.72 \mathrm{keV}$.

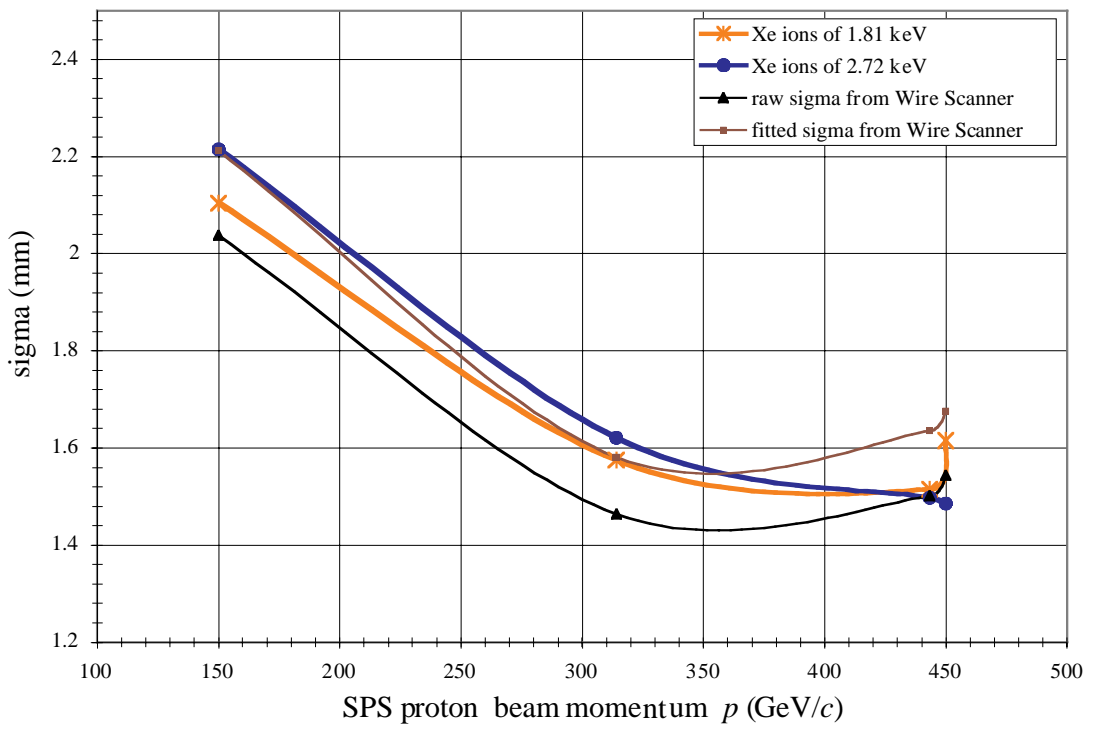

5(c) Evolution of proton-beam horizontal r.m.s. dimension (sigma) as a function of the SPS proton beam momentum; the SPS beam intensity $=1.8 \cdot 10^{13}$ protons. Measurements are made with the wire scanner and with the ion curtain profilometer (after an 'erf fit') using $\mathrm{Xe}^{+}$ions at two different energies. 
Figure $6(\mathrm{~b})$ is a sample of what is observed on a TV display without $[6(\mathrm{~b} .1)]$ and with [6(b.2)] a proton beam. During the SPS acceleration ramp one can qualitatively observe the changes of the ion beam density, which is a consequence of the variation of the proton beam transverse dimension, and also the changes of the proton beam position.

The image obtained every $40 \mathrm{~ms}$ from the camera is processed so as to obtain the ion-beam density with and without the presence of protons. Figure 6(c) shows singleshot measurements at different moments of the SPS cycle, while Fig. 6(d) displays the 'depletion valley range' along the acceleration ramp.

These preliminary measurements are encouraging but still need accurate calibration. This method, though involving a few difficulties in the exact determination of the protonbeam profile, acts as a quasi non-interceptive TV screen.

Fig. 6 Shadowing technique.

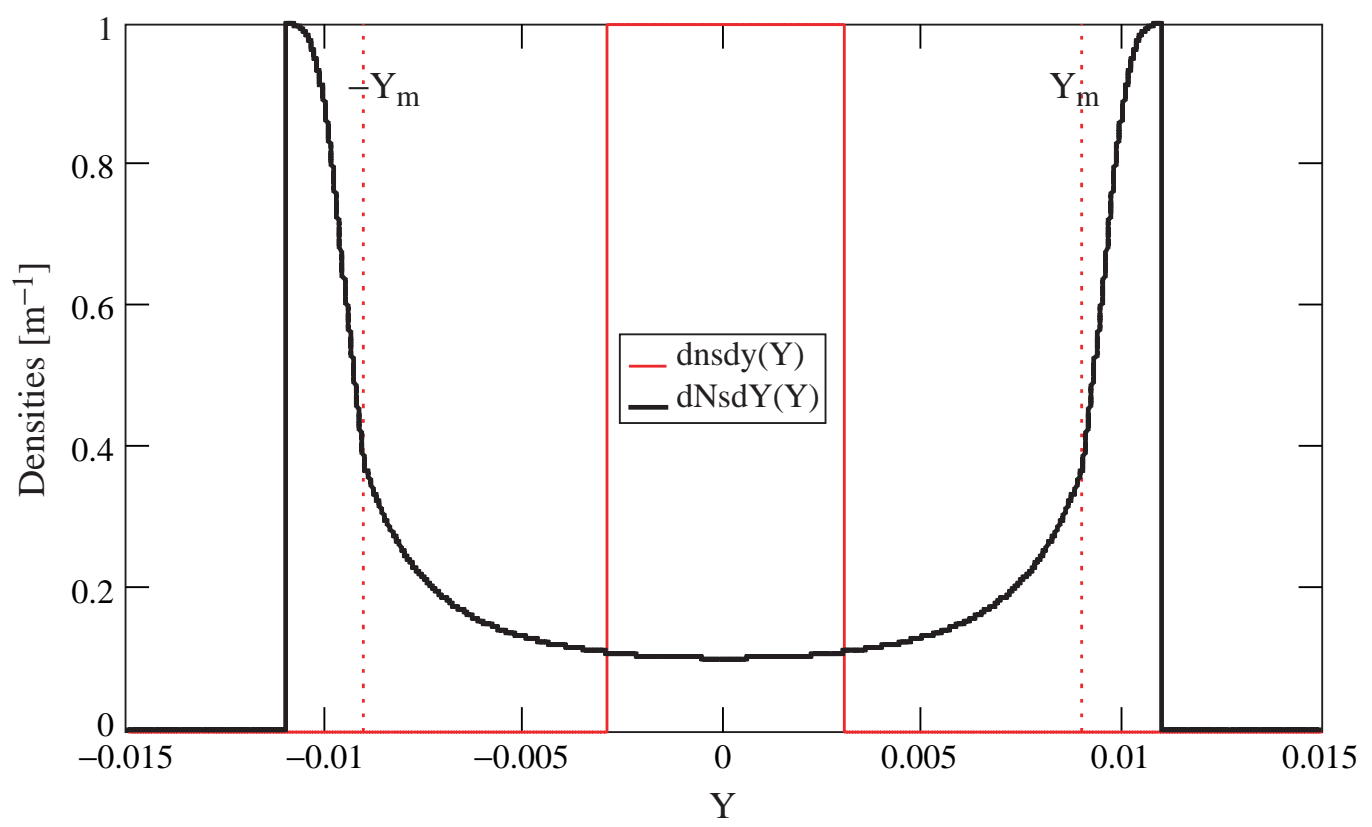

Collector ordinate $[\mathrm{m}]$

Densities versus collector ordinates

6(a) Simulation of the 'shadowing technique'. Horizontal axis: $Y[\mathrm{~m}]$, ion ordinate on the collector. Vertical axis: $\mathrm{d} n s \mathrm{~d} y(Y)$ : ion density $\mathrm{d} n_{\mathrm{i}}(y) / \mathrm{d} y=\mathrm{d} n_{\mathrm{i}}(Y) / \mathrm{d} Y$ on the collector when the proton beam is OFF. $\mathrm{d} N s \mathrm{~d} Y(Y)$ : ion density $\mathrm{d} n_{\mathrm{i}}(Y) / \mathrm{d} Y$ when the proton beam is $\mathrm{ON}$. The vertical lines $\pm Y_{\mathrm{m}}$ correspond to $Y_{\mathrm{m}}=\Delta_{\mathrm{r}}+L \theta\left(\Delta_{\mathrm{r}}\right)$. 


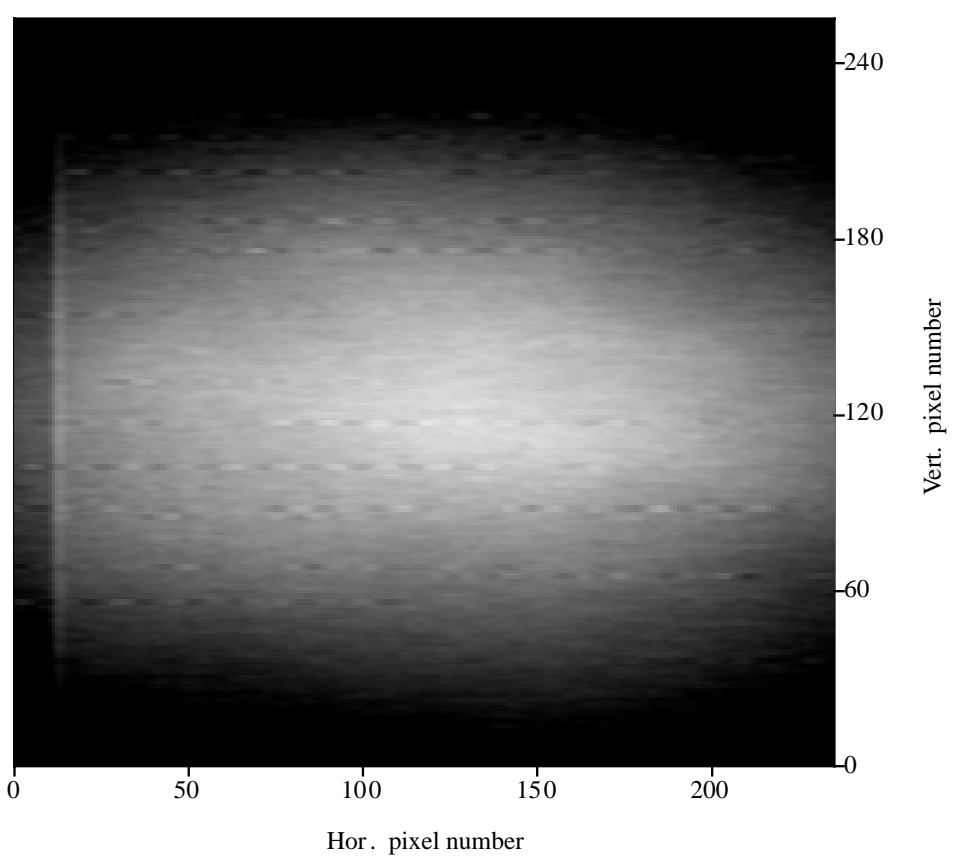

6(b.1) All the experimental measurements are made on the SPS operating in fixed-target mode with $2.8 \cdot 10^{13}$ protons while the ion probe beam consists of $\mathrm{Xe}^{+}$at $3.75 \mathrm{keV}$. Image observed on a TV screen, at a given instant of the SPS cycle when the proton beam is OFF.

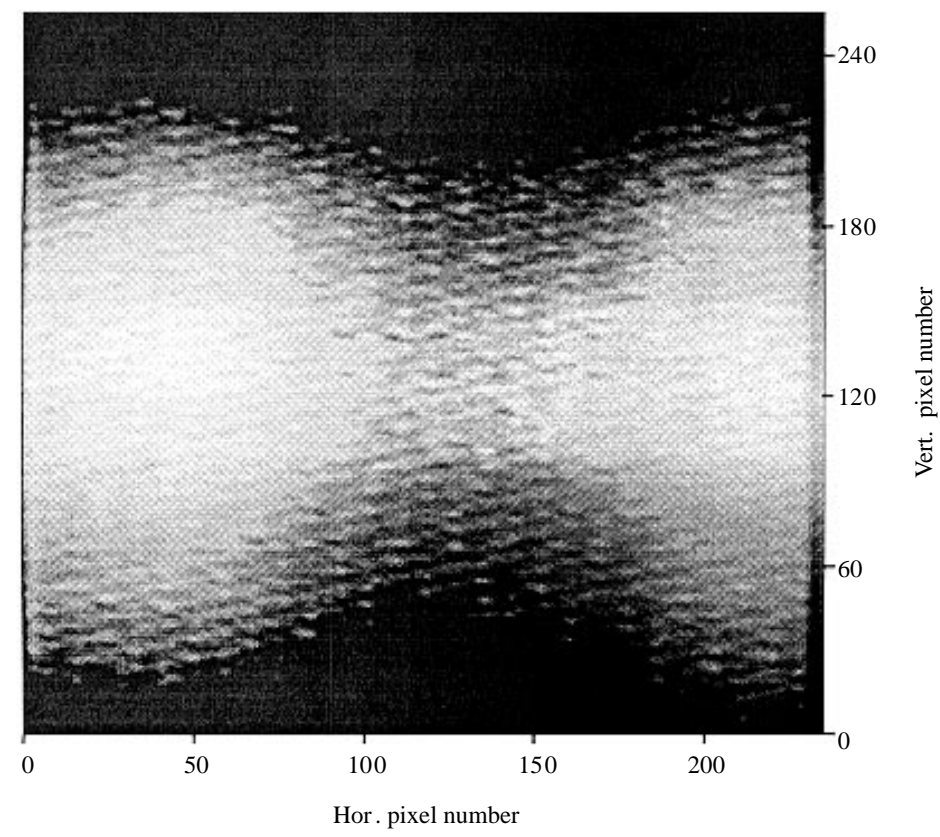

6(b.2) Image observed on a TV screen, at a given instant of the SPS cycle when the proton beam is $\mathrm{ON}$. 
ProfileAT1964

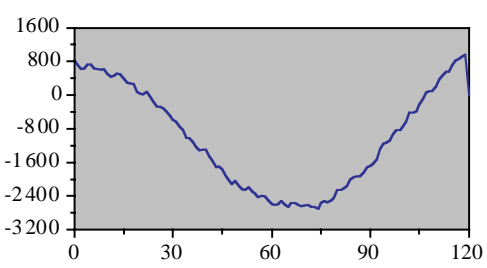

ProfileAT2700

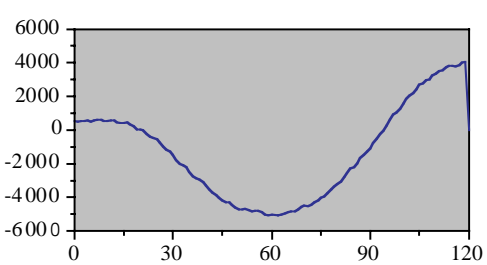

ProfileAT3000

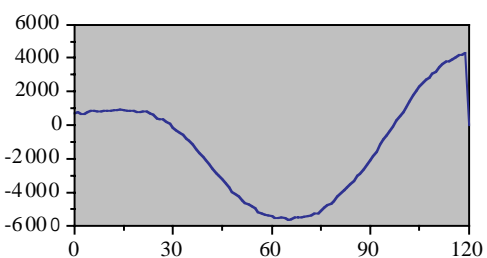

ProfileAT2500

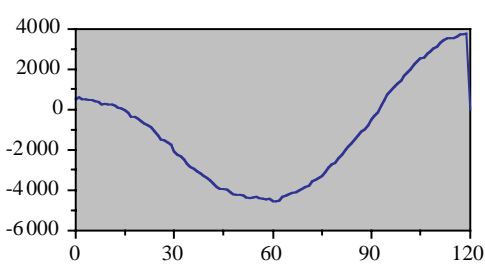

ProfileAT2900

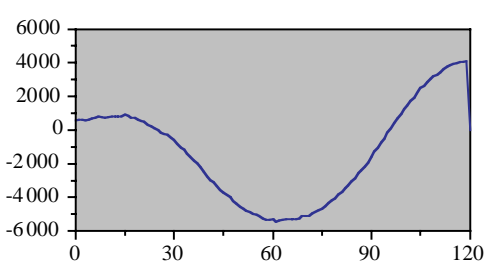

ProfileAT3300

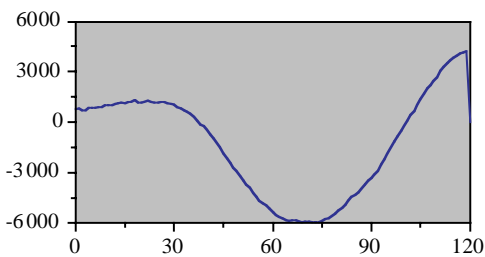

6(c) Depletion on horizontal ion densities observed on the luminescent screen at different instants of the cycle. Horizontal scale: $Y_{0}$ on the collector (arbitrary units, i.e number of pixels). Vertical axis: density in arbitrary units.

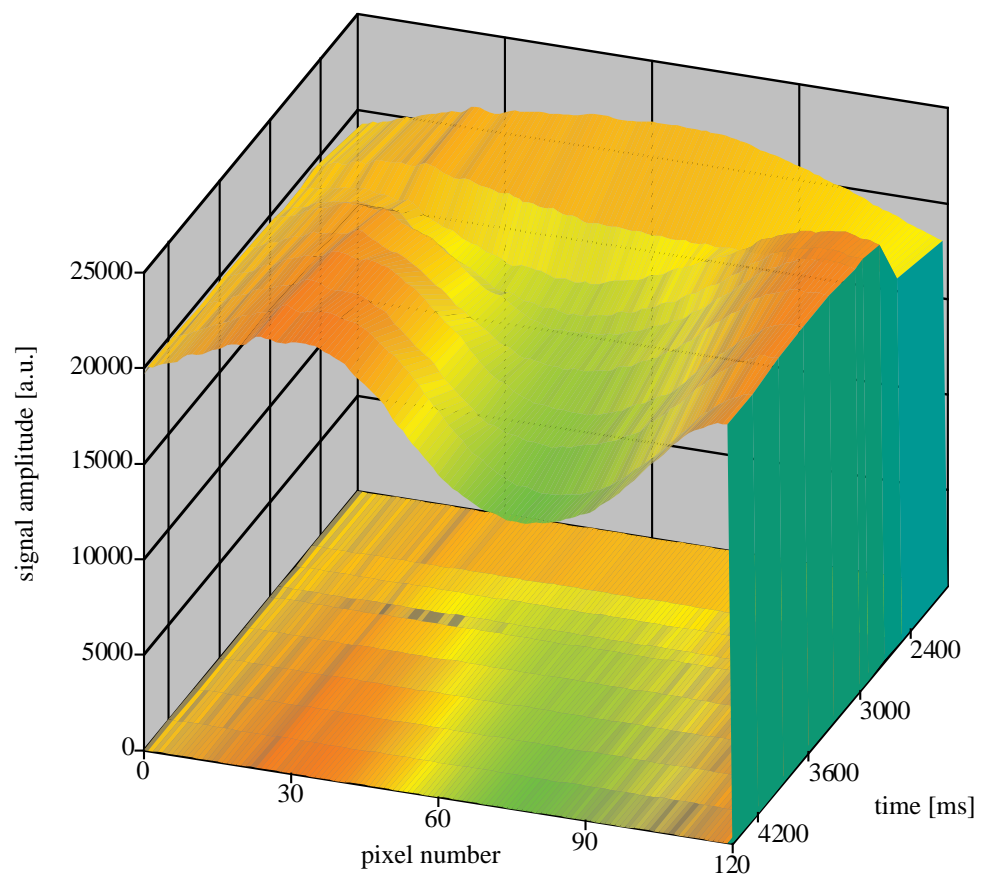

6(d) Valley range distribution versus time. Vertical axis: density in arbitrary units. Horizontal axis: position $Y_{0}$ on the collector (arbitrary units, i.e number of pixels). Longitudinal axis: time in $\mathrm{ms}$. 
This type of detector can be used on other accelerators such as the Linac, Booster, or PS where dense bunches are of interest. The Linac beam parameters and the expected deflection angle are as follows:

beam energy: $0.05 \mathrm{GeV}$,

r.m.s. beam size: $\Delta_{\mathrm{r}}=2.0 \mathrm{~mm}$,

r.m.s. bunch duration: $\sigma_{\mathrm{t}}=0.25 \mathrm{~ns}$,

time interval between bunches: $T=5.0 \mathrm{~ns}$,

beam intensity: $150 \mathrm{~mA} ; n_{\mathrm{b}}=4.64 \cdot 10^{9} \mathrm{p} /$ bunch,

batch duration: 50-100 ms,

maximum expected deflection angle: $\theta_{\max } \cong 20.0 \mathrm{mrad}$.

\subsection{Use on the Linac}

A non-relativistic bunch does not exhibit a purely transverse electric field. Therefore, the longitudinal electric field component has to be taken into account as well. In fact, the longitudinal field at the head of the bunch and that at the end of the bunch are opposite. Simulations [2] show that their effects almost cancel out so that a purely transverse electric field can in practice be considered. An exact analytical demonstration is out of the scope of this paper.

For our test we used the ion pencil-beam technique. As collector we used a resistive rectangular plate whose ends are connected to two low-pass amplifiers, $A$ and $B$. The ion-beam centre of gravity is therefore given by:

$$
\left\langle Y\left(y_{0}\right)\right\rangle=\frac{A-B}{A+B} \cdot \text { constant } .
$$

The present detector is not able to process a single bunch. We thus limit our goal to the measurement of a full batch transverse profile. The pencil beam is therefore scanned through the beam in 50 to $100 \mu \mathrm{s}$. A simulation of the evolution of $\left\langle Y\left(y_{0}\right)\right\rangle$ versus time, depending on whether the proton beam is ON or not, is shown in Fig. 7(a). Experimental measurements are shown on Fig. 7(b) which can be compared with Fig. 7(a). Keeping the pencil beam at a fixed position outside the batch, we measured its maximum deflection angle versus time; thus the intensity variation during the pulse was determined and is shown in Fig. 7(b). The measured batch transverse profile is shown in Fig. 7(c). Since $x(t)\left[\mathrm{m} \cdot \mathrm{s}^{-1}\right]$ is known, one can calibrate the transverse dimension versus time.

It must be emphasized that, for the case of a pencil beam and when using a resistive plate, the measurements can be electronically processed in such a way that the beam profile can be displayed in real time at intervals which are (in the present state of development of our detector) of the order of $50 \mu \mathrm{s}$. 
Fig. 7: Pencil-beam technique using a resistive plate as collector. The position on the collector is determined by the signals $A$ and $B$ measured at both ends of the rectangular resistive plate: $\left\langle Y\left(y_{0}\right)\right\rangle=(A-B) /(A+B)$.

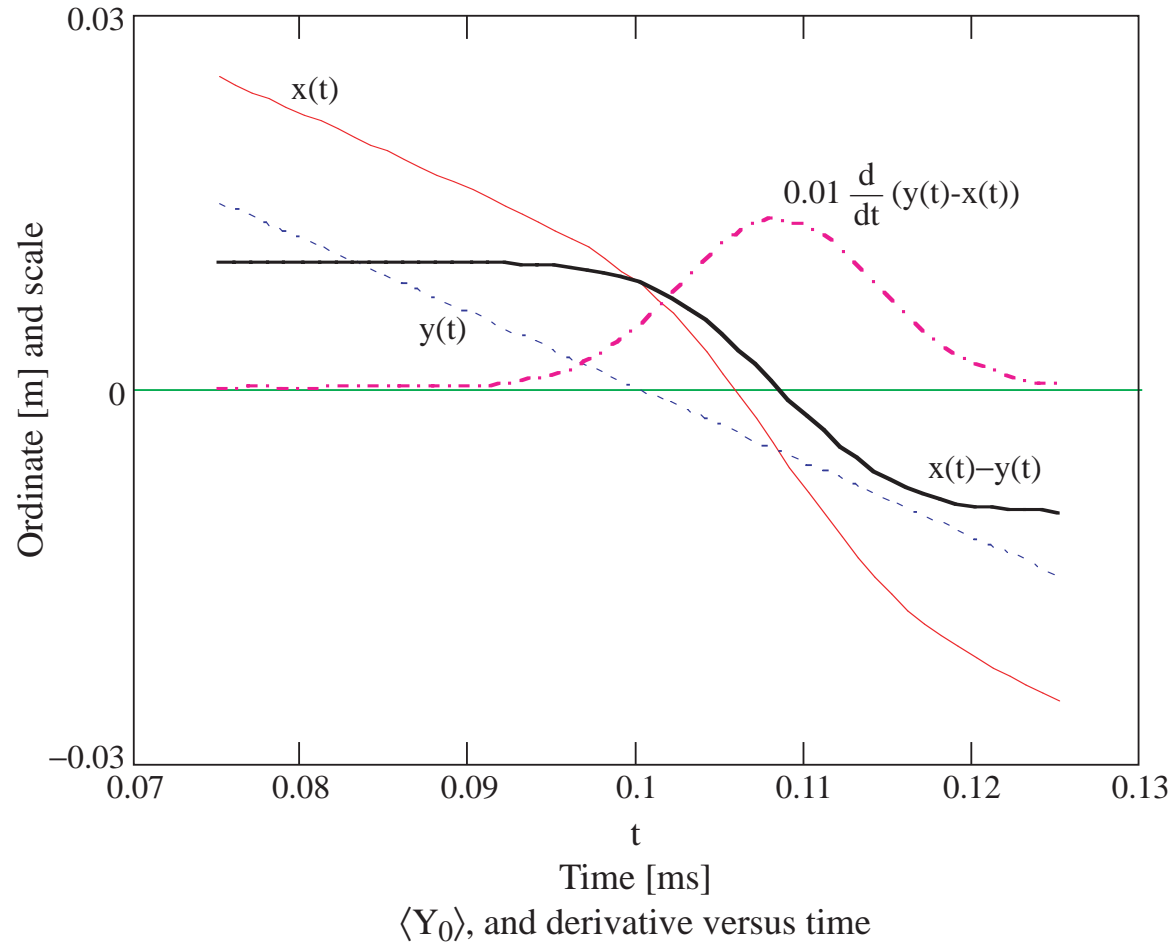

7 (a) Simulations for a proton Gaussian beam. Horizontal axis: time [ms]. Vertical axis: $y(t)=$ $\left\langle Y\left(y_{0}\right)\right\rangle$ when the proton beam is OFF, $x(t)=\left\langle Y\left(y_{0}\right)\right\rangle$ when the proton beam is ON, $(x(t)-$ $y(t)) \equiv L \cdot \theta(t),(\mathrm{d} / \mathrm{d} t)(x(t)-y(t)) \equiv$ proton beam profile.

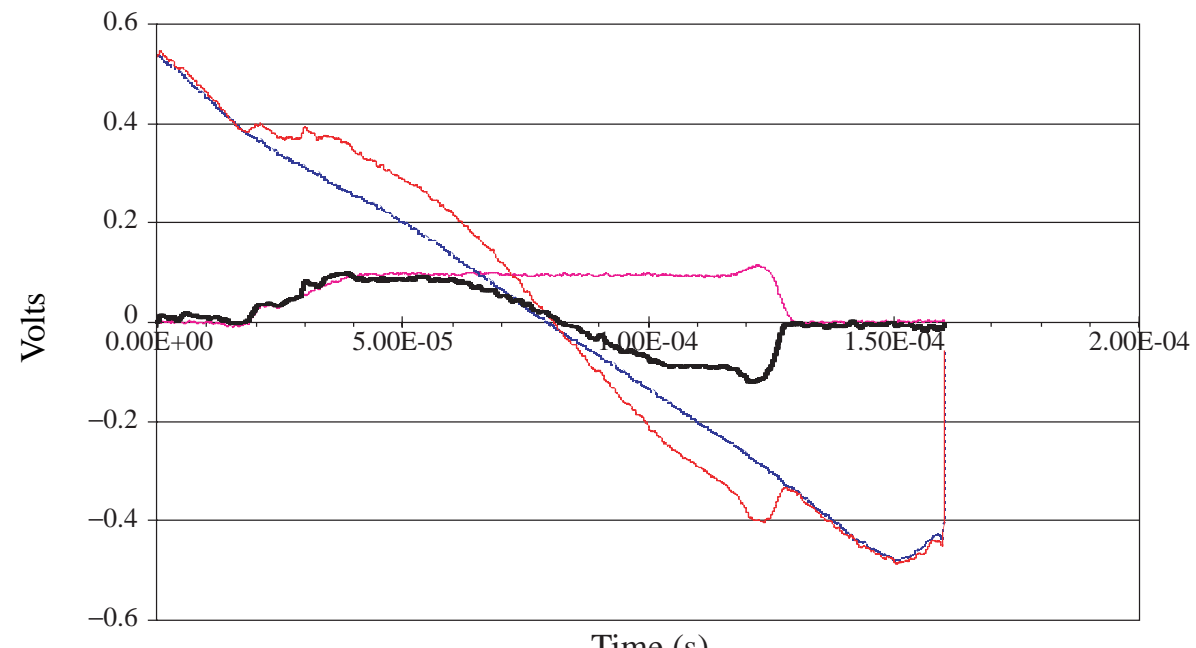

Time (s)

7(b) Measurement of the PS Linac batch. $x(t), y(t), x(t)-y(t)$, can be compared to those of (a). The $(\mathrm{d} / \mathrm{d} t)(x(t)-y(t))$ curve of (a) has been replaced by the representation of the batch intensity, versus time, obtained from the measurement of $\theta_{\max }$. 


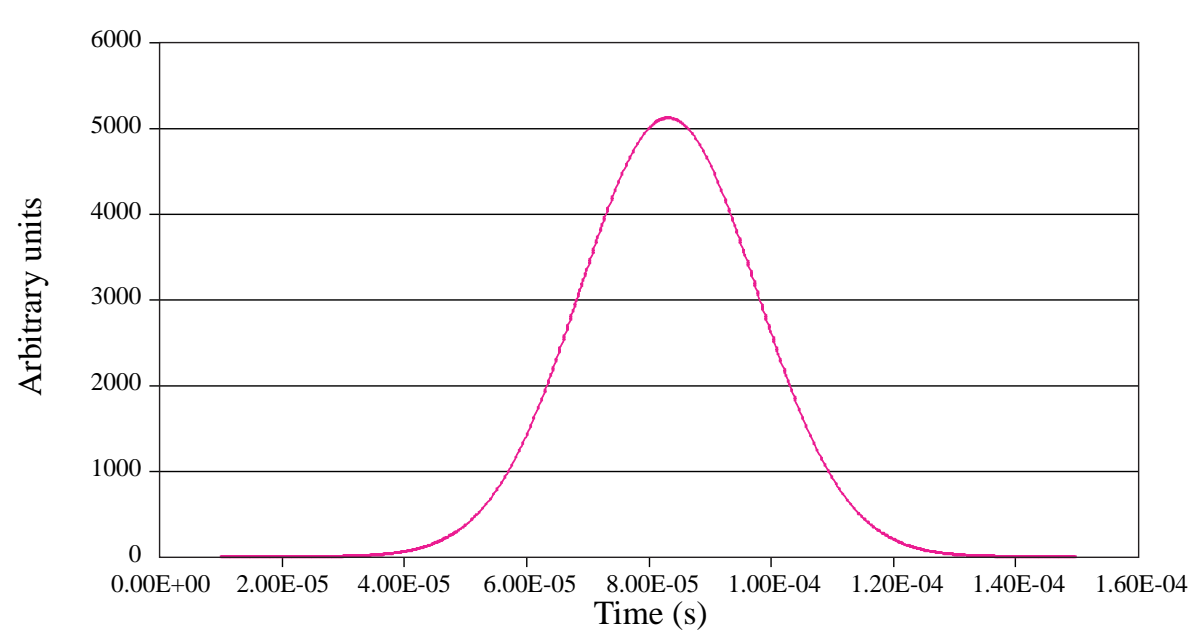

7(c) Batch transverse profile from the measurements shown in (b). From the calibration by means of the well-known $x(t)\left[\mathrm{m} \cdot \mathrm{s}^{-1}\right]$, the transverse r.m.s. dimension is easily determined.

\section{COMMENTS ON ACCURACY AND PROCESSING TIMES}

\subsection{Accuracy}

Let us define by $\sigma_{\text {er }}$ the error introduced by the monitor on the actual beam r.m.s. dimension. The measured r.m.s. dimension is given by: $\sigma_{\mathrm{m}}=\sqrt{\sigma^{2}+\sigma_{\mathrm{er}}^{2}}$, and the relative error by: $(\Delta \sigma / \sigma)=\left(\sigma_{\mathrm{m}}-\sigma\right) / \sigma \cong 1 / 2\left(\sigma_{\mathrm{er}} / \sigma\right)^{2}$. If for example we expect $\Delta \sigma / \sigma=1 / 18$ this would imply that $\sigma_{\text {er }}=\sigma / 3$.

At the SPS where $\sigma$ is of the order of $1 \mathrm{~mm}$ or more, a careful analysis of our measurements showed that:

- when using the pencil-beam technique with $1 \mathrm{~mm}$ width strip foils (spaced by $1.5 \mathrm{~mm}$ ) (Section 7), we estimate $\sigma_{\mathrm{er}}=0.3 \mathrm{~mm}$ and with a resistive plate (Section 10.1) $\sigma_{\mathrm{er}}=0.1 \mathrm{~mm}$;

- when using the curtain beam with a CCD camera (Section 8) we estimate $\sigma_{\text {er }}=$ $0.1 \mathrm{~mm}$ (the accuracy being 1 pixel and the resolution $0.1 \mathrm{~mm} /$ pixel);

- in the future the use of strips spaced at $0.2 \mathrm{~mm}$ would reduce the error made on the r.m.s. dimension measurement to $0.1 \mathrm{~mm}$. Concerning the use of image detectors, a conservative approach is to keep $0.1 \mathrm{~mm} /$ pixel. It must be kept in mind that the collector has to cope not only with the proton beam size but also with its displacement in time. The relative displacement reduces the r.m.s. measurement accuracy.

\subsection{Processing time}

With strips or resistive plates, the time interval between measured profiles is presently limited to $50 \mu \mathrm{s}$. Attempts are under way to reduce this time to $5 \mu \mathrm{s}$ (it will also depend on the available source intensity). For light monitors, the standard processing time is presently $40 \mathrm{~ms}$. Further reduction of this large time interval will depend on technological progress.

\section{CONCLUSION}

The proofs of principle of the three methods, namely the first using an ion pencilbeam, the second using a curtain beam, and the third making use of the shadowing 
technique, have been experimentally demonstrated. The ion source quality has to be improved as well as the electronic and processing techniques. With an improved detector, we can expect to measure profiles every $5 \mu \mathrm{s}$ with an error on the r.m.s. dimension of the order of $50 \mu \mathrm{m}$ for LHC-type beams.

\section{Acknowledgements}

We want to thank G. Ferioli, G. Arduini and the SPS Vacuum team for their efficient help during our experiment. We also acknowledge the competence of the CERN Desktop Publishing Service in the drafting of this paper.

\section{References}

[1] (a) J. Bosser and I. Meshkov, Profilometer for small dimension proton beam, CERNPS/BD/Note 94-04, CERN, 7 March 1994. (b) W. Nexsen et al., Minimal interference beam size/profile measurement technique applicable to the collider, Superconducting Super Collider Laboratory, SSCL-631, May 1993. (c) E. Tsyganov et al., Electron beam emittance monitor for the SSC, in Proc. 1993 Particle Accelerator Conference, Washington (IEEE, Piscataway, N.J., 1994), Vol. 3, pp. 2489-2491.

[2] (a) J. Bosser, A. Feschenko and R. Maccaferri, Ion profilometer for the SPS and LHC accelerators, CERN-PS/BD/Note 99-07, CERN, September 1999. An exhaustive list of relevant papers is given in this reference. (b) J.A. Pasour and Mai T. Ngo, Ion probe beam position and profile measurement, in Proc. 6th Workshop on Beam Instrumentation, Vancouver, 1994, Eds. G.H. Mackenzie, Bill Rawnsley and J. Thomson, AIP Conference Proceedings No. 333, (AIP, Woodbury, NY, 1995).

[3] J. Bosser, E. Chevallay, C. Dimopoulou, A. Feschenko and R. Maccaferri, Ion curtain profilometer, CERN-PS/BD/Note 99-15, CERN, 1999.

[4] C. Bovet (CERN), private communication. 


\section{APPENDIX A}

We aim to demonstrate that the deviation angle $\theta\left(y_{0}\right)$ can, under some assumptions, be approximated by an error function. We use the symbols of Section 2.3 and those of Fig. 1(b).

\section{HYPothesis}

We consider a proton bunch having

- a transverse normalized distribution

$$
n_{\perp}(r)=\frac{2}{2 \pi \Delta_{\mathrm{r}}^{2}} \exp \left(-\frac{r^{2}}{\Delta_{\mathrm{r}}^{2}}\right), \quad r=\sqrt{x^{2}+y^{2}}
$$

with r.m.s. value: $\left(\int_{0}^{\infty} r^{2} n_{\perp}(r) 2 \pi r \mathrm{~d} r\right)^{1 / 2}=\Delta_{\mathrm{r}}$

- a uniform longitudinal distribution over the length $L_{\mathrm{b}}=\sqrt{2 \pi} \Delta_{\mathrm{s}}$ so that the normalized longitudinal distribution $n_{\sigma}(z)=1 / L_{\mathrm{b}}$.

So the overall distribution is

$$
n(r)=n_{\mathrm{b}} \cdot \frac{1}{L_{\mathrm{b}}} \cdot \frac{2}{2 \pi \Delta_{r}^{2}} \exp \left(-\frac{r^{2}}{\Delta_{\mathrm{r}}^{2}}\right),
$$

where $n_{\mathrm{b}} \equiv$ number of protons/bunch.

DEFlECtion ANGLE AS A FUnCtion OF THE IMPACT PARAMETER $y_{0}$

It has been shown [1] that

$$
\theta\left(y_{0}\right)=\frac{Q V_{0}}{2 E_{\mathrm{k}}} \int_{-u_{\mathrm{i}}}^{u_{\mathrm{f}}} \frac{1-\exp \left[-\frac{y_{0}^{2}}{\Delta_{\mathrm{r}}^{2}}\left(1+u^{2}\right)\right]}{1+u^{2}} \mathrm{~d} u
$$

$u \equiv x / y_{0}, u_{\mathrm{f}} \equiv x_{\mathrm{f}} / \Delta_{\mathrm{r}} \gg 1, u_{\mathrm{i}} \equiv x_{\mathrm{i}} / \Delta_{\mathrm{r}} \gg 1$ where the symbols are those defined in Sections 2 and 3. Differentiating:

$$
\frac{\mathrm{d} \theta\left(y_{0}\right)}{\mathrm{d} y_{0}}=\frac{Q V_{0}}{2 E_{\mathrm{k}}} \frac{2 y_{0}}{\Delta_{\mathrm{r}}^{2}} \exp \left(-\frac{y_{0}^{2}}{\Delta_{\mathrm{r}}^{2}}\right) \int_{-u_{\mathrm{i}}}^{u_{\mathrm{f}}} \exp \left[-\left(\frac{u y_{0}}{\Delta_{\mathrm{r}}}\right)^{2}\right] \mathrm{d} u
$$

and for $u_{\mathrm{f}}, u_{\mathrm{i}} \rightarrow \infty$ a good approximation will be

$$
\left(\frac{\mathrm{d} \theta\left(y_{0}\right)}{\mathrm{d} y_{0}}\right)_{\text {approx }}=\frac{Q V_{0}}{2 E_{\mathrm{k}}} \cdot \frac{2 \sqrt{\pi}}{\Delta_{\mathrm{r}}} \exp \left(-\frac{y_{0}^{2}}{\Delta_{\mathrm{r}}^{2}}\right) .
$$

\section{ERROR FUNCTION}

It is well known that

$$
\operatorname{erf}(u) \equiv \frac{2}{\sqrt{\pi}} \int_{0}^{u} \exp \left(-\xi^{2}\right) \mathrm{d} \xi, \quad \frac{\mathrm{d}(\operatorname{erf}(u))}{\mathrm{d} u}=\frac{2}{\sqrt{\pi}} \exp \left(-u^{2}\right),
$$

$\operatorname{erf}(0)=0, \operatorname{erf}(\infty)=1, \operatorname{erf}(x)=-\operatorname{erf}(-x)$

When applied to Eq. (A.3) this gives an approximate expression (A.4) 


$$
\theta_{\text {approx }}\left(y_{0}\right)=\frac{Q V_{0}}{2 E_{\mathrm{k}}} \pi \operatorname{erf}\left(\frac{y_{0}}{\Delta_{\mathrm{r}}}\right)+C
$$

with the constant $C=0$ since $\theta(0)=0$.

We set the maximum deviation angle

$$
\theta_{\max }=\frac{Q V_{0}}{2 E_{\mathrm{k}}} \cdot \pi
$$

and are thus in position to summarize:

$$
\begin{gathered}
\theta_{\text {approx }}\left(y_{0}\right)=\theta_{\max } \operatorname{erf}\left(\frac{y_{0}}{\Delta_{\mathrm{r}}}\right) \\
\left(\frac{\mathrm{d} \theta\left(y_{0}\right)}{\mathrm{d} y_{0}}\right)_{\text {approx }}=\frac{2 \theta_{\max }}{\sqrt{\pi} \cdot \Delta_{\mathrm{r}}} \exp \left(-\frac{y_{0}^{2}}{\Delta_{\mathrm{r}}^{2}}\right) \\
\Delta_{\mathrm{r}}=\frac{2 \theta_{\max }}{\sqrt{\pi}} \frac{1}{\left.\frac{\mathrm{d} \theta}{\mathrm{d} y_{0}}\right|_{y_{0}} \cong 0} .
\end{gathered}
$$

\section{ANOTHER EXPRESSION OF THE TRANSVERSE R.M.S. VALUE}

Keeping the hypothesis of a Gaussian proton bunch:

- $n_{\perp}(r)$ is a two-dimensional Gaussian distribution $\left(r^{2}=x^{2}+y^{2}\right)$ with r.m.s. value

$-\left(\frac{\mathrm{d} \theta\left(y_{0}\right)}{\mathrm{d} y_{0}}\right)_{\text {approx }}$ is a one-dimensional Gaussian distribution function of $y_{0}$ whose r.m.s. value is computed as follows:

$$
\left[\frac{\int_{-\infty}^{\infty}\left(\frac{\mathrm{d} \theta\left(y_{0}\right)}{\mathrm{d} y_{0}}\right)_{\text {approx }} y_{0}^{2} \mathrm{~d} y_{0}}{\int_{-\infty}^{\infty}\left(\frac{\mathrm{d} \theta\left(y_{0}\right)}{\mathrm{d} y_{0}}\right)_{\text {approx }} \mathrm{d} y_{0}}\right]^{1 / 2}=\frac{\Delta_{\mathrm{r}}}{\sqrt{2}} .
$$

It is convenient to set $\sigma \equiv \Delta_{\mathrm{r}} / \sqrt{2}$ and to write instead

$$
\begin{gathered}
\theta_{\text {approx }}\left(y_{0}\right)=\theta_{\max } \operatorname{erf}\left(\frac{y_{0}}{\sqrt{2} \cdot \sigma}\right) \\
\left(\frac{\mathrm{d} \theta\left(y_{0}\right)}{\mathrm{d} y_{0}}\right)_{\text {approx }}=2 \theta_{\max } \frac{1}{\sqrt{2 \pi} \cdot \sigma} \exp \left(-\frac{y_{0}^{2}}{2 \sigma^{2}}\right), \\
\sigma=\frac{1}{\sqrt{2 \pi}} 2 \theta_{\max } \frac{1}{\left.\frac{\mathrm{d} \theta}{\mathrm{d} y_{0}}\right|_{y_{0} \cong 0}} .
\end{gathered}
$$




\section{APPENDIX B}

For practical reasons, the $\mathrm{Xe}^{+}$beam coming out of the source might have a conical distribution. This ion beam is collimated, steered, and focused in order to obtain the ion curtain. Therefore, not only do the probe ions move in the $x$ direction but they also have a velocity component lying in the $y z$ plane.

Figure B.1 shows the projection of the probe ion trajectory in the $x y$ plane and Fig. B.2 shows the projection of the probe ion trajectory in the $x z$ plane. The parameters $D=916 \mathrm{~mm}$ and $L=400 \mathrm{~mm}$.

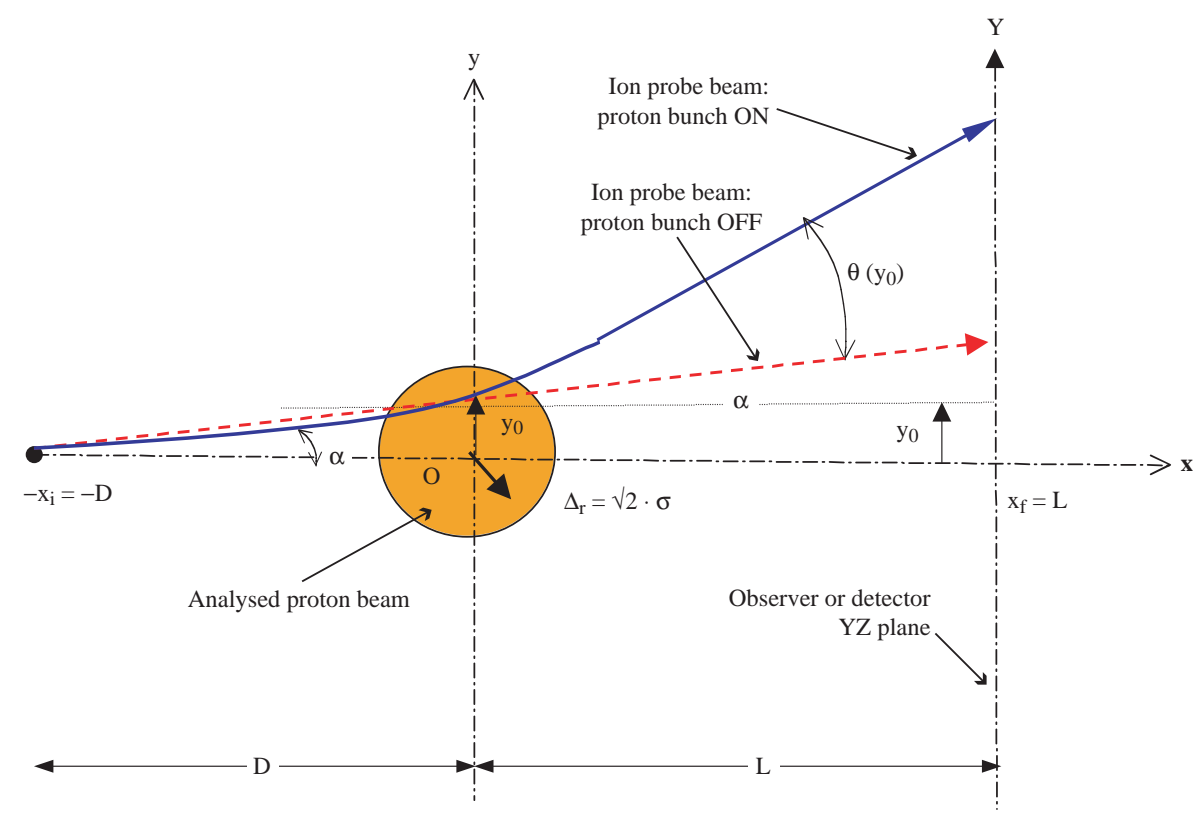

Fig. B.1

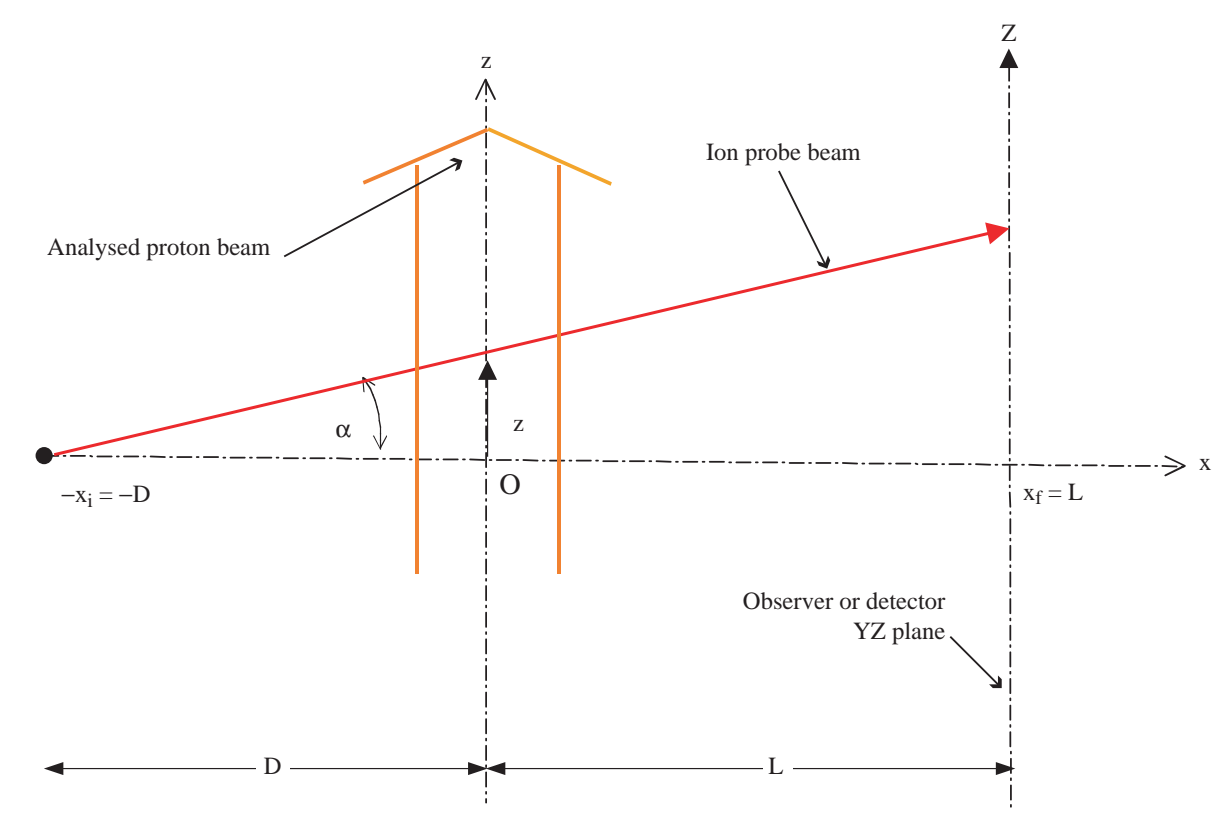

Fig. B.2 
With the proton bunch OFF, the probe ion reaches the observer plane (Fig. B.1) at $Y=(L+D) \cdot \operatorname{tg} \alpha \cong(L+D) \cdot \frac{y_{0}}{D}=\left(1+\frac{L}{D}\right) \cdot y_{0}$, whereas with the proton bunch ON the probe ion is also deflected by an angle $\theta\left(y_{0}\right)$ and hence reaches the observer plane at $Y=\left(1+\frac{L}{D}\right) \cdot y_{0}+L \cdot \operatorname{tg} \theta\left(y_{0}\right) \cong\left(1+\frac{L}{D}\right) \cdot y_{0}+L \cdot \theta\left(y_{0}\right)$.

In both cases the probe ion drifts in the $z$ direction (Fig. B.2) and reaches the observer plane at $Z=\left(1+\frac{L}{D}\right) \cdot z$.

This geometrical set-up was taken into account in the treatment of experimental data. However, in order to simplify the notations, throughout the paper we considered the ion source to be very far away from the probed beam $(D \rightarrow \infty)$ so that the $y, z$ velocity components of the ions are practically negligible and $1+\frac{L}{D} \cong 1$. 


\section{APPENDIX C}

We aim to analyse in more detail the theoretical aspects of the shadowing technique.

As already said, the ion distribution on the observer plane is expressed by

$$
\frac{\mathrm{d} n_{\mathrm{i}}}{\mathrm{d} Y}=\frac{\mathrm{d} n_{\mathrm{i}}}{\mathrm{d} y} \frac{\mathrm{d} y}{\mathrm{~d} Y}
$$

where $\mathrm{d} n_{\mathrm{i}} / \mathrm{d} y$ is the ion distribution measured when the proton beam is OFF.

We have

$$
Y=y+L \theta(y) \equiv g(y) \quad \text { or } \quad y=g^{-1}(Y) .
$$

From Eqs. (2a) and (2d):

$$
\begin{gathered}
\theta(y)=\theta_{\max } \operatorname{erf}\left(\frac{y}{\Delta_{\mathrm{r}}}\right) \\
\frac{\mathrm{d} \theta(y)}{\mathrm{d} y}=\frac{2 \theta_{\max }}{\sqrt{\pi} \cdot \Delta_{\mathrm{r}}} \exp \left(-\frac{y^{2}}{\Delta_{\mathrm{r}}^{2}}\right)
\end{gathered}
$$

The function $g(y)$ itself is not simple and therefore $g^{-1}$ is not straightforward.

Since

$$
\frac{\mathrm{d}\left(g^{-1}(Y)\right)}{\mathrm{d} Y}=\frac{1}{\left.\frac{\mathrm{d} g}{\mathrm{~d} y}\right|_{y=g^{-1}(Y)}}
$$

and

$$
\frac{\mathrm{d} g(y)}{\mathrm{d} y}=1+L \cdot \frac{2 \theta_{\max }\left(n_{\mathrm{b}}\right)}{\sqrt{\pi} \cdot \Delta_{\mathrm{r}}} \exp \left(-\frac{y^{2}}{\Delta_{\mathrm{r}}^{2}}\right)
$$

we obtain

$$
\frac{\mathrm{d}\left(g^{-1}(Y)\right)}{\mathrm{d} Y}=\frac{\mathrm{d} y}{\mathrm{~d} Y}(Y)=\frac{1}{1+L \cdot \frac{2 \theta_{\max }\left(n_{\mathrm{b}}\right)}{\sqrt{\pi} \cdot \Delta_{\mathrm{r}}} \exp \left(-\left(\frac{g^{-1}(Y)}{\Delta_{\mathrm{r}}}\right)^{2}\right)}
$$

remembering that $\theta_{\max }$ depends on $n_{\mathrm{b}}$ and therefore on the proton beam intensity.

To illustrate this effect let us consider a simplified expression instead of an error function:

$$
\begin{aligned}
& \theta(y)= \begin{cases}\theta_{\max }\left(n_{\mathrm{b}}\right) \cdot \frac{y}{\Delta_{\mathrm{r}}} & \text { for }|y| \leq 2 \Delta_{\mathrm{r}} \\
\theta_{\max }\left(n_{\mathrm{b}}\right) & \text { for }|y|>2 \Delta_{\mathrm{r}}\end{cases} \\
& g(y)=Y(y)=\left\{\begin{array}{lll}
y+L \cdot \theta_{\max }\left(n_{\mathrm{b}}\right) \cdot \frac{y}{\Delta_{\mathrm{r}}} & \text { for } & |y| \leq 2 \Delta_{\mathrm{r}} \\
y+L \cdot \theta_{\max }\left(n_{\mathrm{b}}\right) & \text { for } & |y|>2 \Delta_{\mathrm{r}}
\end{array}\right.
\end{aligned}
$$

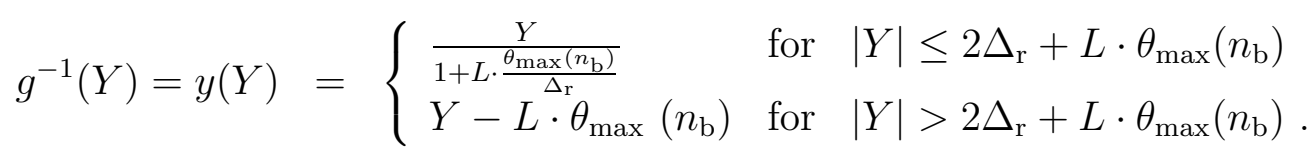


Then we have:

$$
\frac{\mathrm{d} n_{\mathrm{i}}}{\mathrm{d} Y}=\frac{\mathrm{d} n_{\mathrm{i}}}{\mathrm{d} y}\left\{\begin{array}{cll}
\frac{1}{1+L \cdot \frac{\theta_{\max \left(n_{\mathrm{b}}\right)}}{\Delta_{\mathrm{r}}}} & \text { for } & |Y| \leq 2 \Delta_{\mathrm{r}}+L \cdot \theta_{\max }\left(n_{\mathrm{b}}\right) \\
1 & \text { for } & |Y|>2 \Delta_{\mathrm{r}}+L \cdot \theta_{\max }\left(n_{\mathrm{b}}\right) .
\end{array}\right.
$$

Figure C.1 shows the curves $\mathrm{d} n_{\mathrm{i}} / \mathrm{d} Y$ as a function of $Y$ for $\Delta_{\mathrm{r}}=10^{-3} \mathrm{~m}, L=0.5 \mathrm{~m}$ and for two different values of $\theta_{\max }$, namely for $\theta_{\max }=5 \times 10^{-3} \mathrm{rad}$ (curve $\mathrm{d} n_{\mathrm{i}} 1 / \mathrm{d} Y$ on the plot) and for $\theta_{\max }=10 \times 10^{-3} \mathrm{rad}$ (curve $\mathrm{d} n_{\mathrm{i}} 2 / \mathrm{d} Y$ on the plot). We considered only the simple case in which the initial ion curtain distribution is uniform, that is $\left(\mathrm{d} n_{\mathrm{i}} / \mathrm{d} y\right)=$ const $=1$.

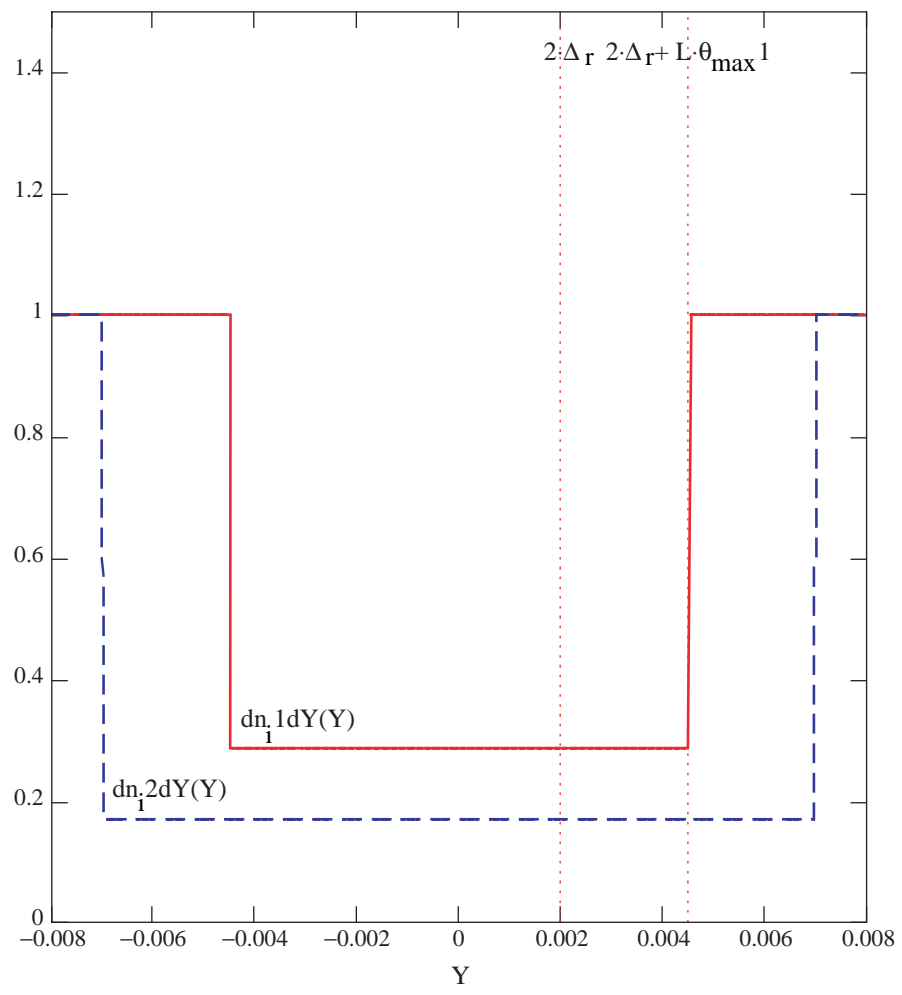

Fig. C.1

We see that the depletion width on the luminescent screen is $2\left(2 \Delta_{\mathrm{r}}+L \theta_{\max }\left(n_{\mathrm{b}}\right)\right)$. The discontinuity of the curve occurs at $Y=\left(2 \Delta_{\mathrm{r}}+L \theta_{\max }\left(n_{\mathrm{b}}\right)\right)$ and will remain at this point if $\Delta_{\mathrm{r}}$ and $\theta_{\max }$ both vary in such a way that $\mathrm{d}(Y)=\mathrm{d}\left(2 \Delta_{\mathrm{r}}+L \theta_{\max }\left(n_{\mathrm{b}}\right)\right)=0$, or $2 \mathrm{~d}\left(\Delta_{\mathrm{r}}\right)=\operatorname{Ld}\left(\theta_{\max }\left(n_{\mathrm{b}}\right)\right)$, i.e. the r.m.s. proton beam size variation $\mathrm{d}\left(\Delta_{\mathrm{r}}\right)$ is compensated by a variation of $\theta_{\max }$ due to an increase of bunch intensity $\left(n_{\mathrm{b}}\right)$ and vice versa. 